\title{
Imaging of all three coronary arteries by transthoracic echocardiography. an illustrated guide Marek Krzanowski*1, Wojciech Bodzon ${ }^{1}$ and Paweł Petkow Dimitrow ${ }^{2}$
}

Address: ${ }^{1}$ The Department of Medicine, Jagiellonian University School of Medicine, 8 Skawińska Str., Kraków, Poland and 22nd Department of Cardiology, Jagiellonian University School of Medicine, 17 Kopernika Str., Kraków, Poland

Email: Marek Krzanowski* - mkrzano@mp.pl; Wojciech Bodzoń - wbodzon@mp.pl; Paweł Petkow Dimitrow - dimitrow@mp.pl

* Corresponding author

Published: 17 November 2003

Cardiovascular Ultrasound 2003, I:16
Received: 03 September 2003

Accepted: 17 November 2003

This article is available from: http://www.cardiovascularultrasound.com/content/l/I/16

(C) 2003 Krzanowski et al; licensee BioMed Central Ltd. This is an Open Access article: verbatim copying and redistribution of this article are permitted in all media for any purpose, provided this notice is preserved along with the article's original URL.

\begin{abstract}
Background: Improvements in ultrasound technology has enabled direct, transthoracic visualization of long portions of coronary arteries : the left anterior descending (LAD), circumflex (Cx) and right coronary artery (RCA). Transthoracic measurements of coronary flow velocity were proved to be highly reproducible and correlated with invasive measurements. While clinical applications of transthoracic echocardiography (TTE) of principal coronary arteries are still very limited they will likely grow. The echocardiographers may therefore be interested to know the ultrasonic views, technique of examination and be aware where to look for coronary arteries and how to optimize the images.
\end{abstract}

Methods: A step-by-step approach to direct, transthoracic visualization of the LAD, Cx and RCA is presented. The technique of examination is discussed, correlations with basic coronary angiography views and heart anatomy are shown and extensively illustrated with photographs and movie-pictures. Hints concerning optimization of ultrasound images are presented and artifacts of imaging are discussed.

Conclusions: Direct, transthoracic examination of the LAD, Cx and RCA in adults is possible and may become a useful adjunct to other methods of coronary artery examination but studies are needed to establish its role.

\section{Background}

Improvements in ultrasound machines and introduction of the harmonic imaging and the ultrasound contrast agents into clinical practice have enabled direct, transthoracic visualization of long portions of coronary arteries : the left anterior descending (LAD), circumflex (Cx) and right coronary artery (RCA). Most of the studies has dealt with assessment of the middle and distal LAD which can now be visualized in almost every patient [1-8] or the pos- terior interventricular branch, which in skilful hands can be assessed in 3/4 of patients [9]. We have previously shown, that both Cx and RCA can be successfully assessed in a considerable number of sequential adults scheduled for coronary angiography $[10,11]$ and it is known, that they can be studied in children $[12,13]$. Transthoracic measurements of coronary flow velocity were proved to be highly reproducible and correlated with invasive measurements [1,3,14-16]. While clinical applications of 


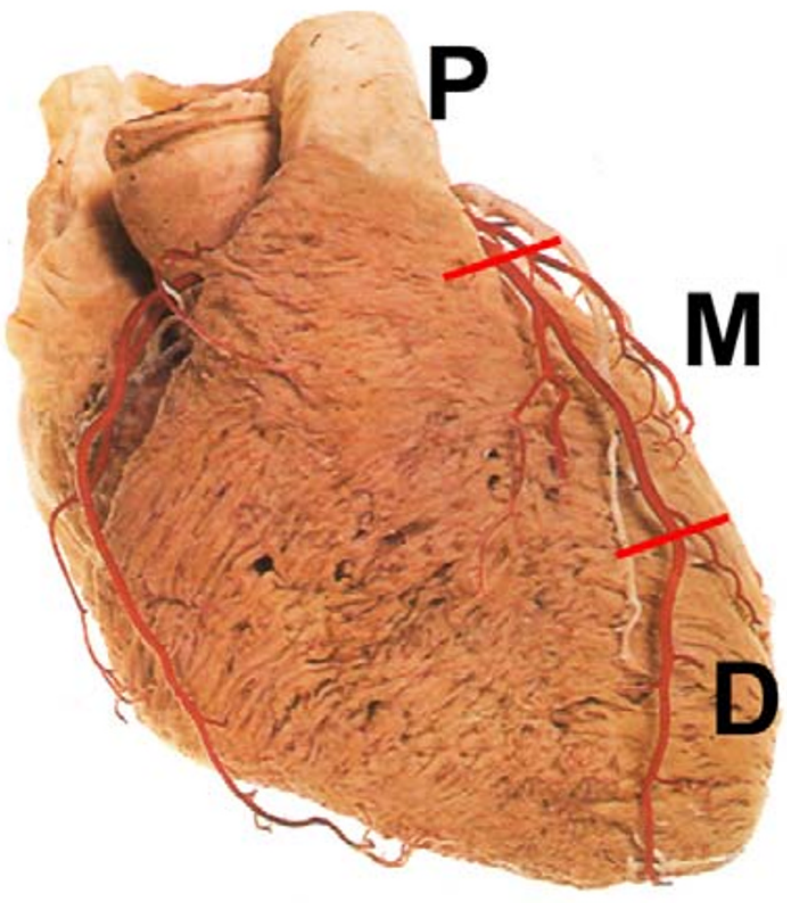

Figure I

An artists drawing of the heart, right antero-oblique (RAO) view. Division of left anterior descending (LAD) coronary artery into segments: proximal $(P)$, middle $(M)$ and distal $(D)$.

transthoracic echocardiography in direct assessment of coronary arteries are very limited they are likely to grow. The echocardiographers may, therefore, be interested to know the ultrasonic views, technique of examination and ways of optimizing the images.

\section{Aims of the article}

The primary aims of the article, which is published under the heading "technical note" are: 1 . to provide the practical description of trans-thoracic approach to coronary artery visualization and detection of the velocity profile in the coronary arteries, 2 . to present basic transthoracic echocardiographic views of coronary arteries: the LAD, Cx and RCA, 3. to suggest a method of their division into segments. The secondary aim of the article is to describe a single centre experience with transthoracic visualization of coronary arteries. It is hoped that the paper will help to expand this interesting and promising technique.

\section{Methods}

We suggest to divide each coronary artery into three segments of approximately the same length (see Figs. $1,2,3,4,5$, table 1 ). The division does not, however, follow



Figure 2

Angiography of the left coronary artery in RAO projection with caudal angulation. Division of LAD into segments: proximal $(P)$, middle $(M)$ and distal $(D)$.

exact angiographic classification. The differences are most pronounced with regard to the RCA. As the origin of the circumflex coronary artery cannot always be localized, the left main coronary artery and the proximal part of the left anterior descending artery form a common segment.

A modern, high quality ultrasound machine is required for successful scanning. In most transthoracic, echocardiographic examinations (TTE) performed in our laboratory we have used an Acuson Sequoia 512 ultrasound instrument (Acuson Corp. Mountainview, Ca). We are using either 5.0 or $3.5 \mathrm{MHz}$ narrow-band sector transducers in second harmonic mode for B-mode examination while both color Doppler coronary artery mapping and spectral Doppler coronary flow velocity assessment are performed at 2.5 or $2 \mathrm{MHz}$. The coronary arteries can, however, be imaged with ultrasound instruments made by other manufacturers and with both broadband and high frequency transducers.

We believe that the harmonic imaging considerably facilitates the examination. Many investigators $[1,3,4,17]$ but not all [11] concluded, that ultrasound contrast agents provided a substantial improvement in the quality of Doppler coronary artery images. While technology of 


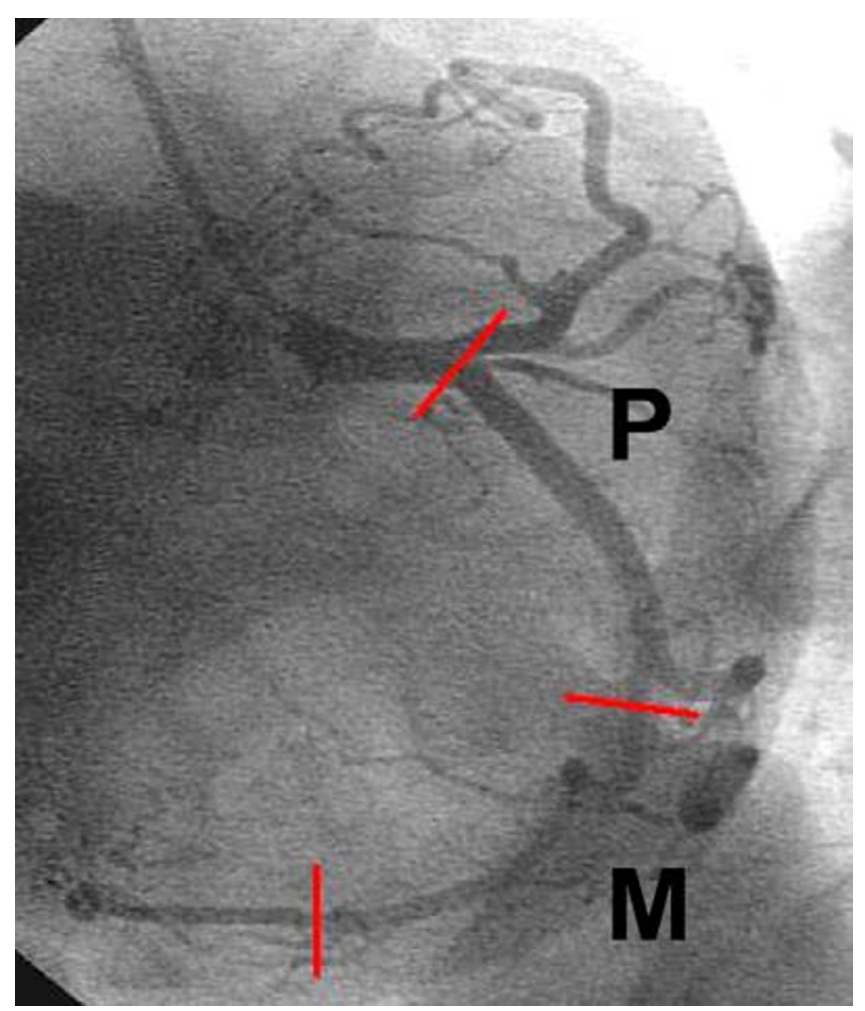

Figure 3

Angiography of the left coronary artery in left antero-oblique (LAO) projection with caudal angulation. Division of left circumflex $(\mathrm{Cx})$ coronary artery into segments: proximal $(\mathrm{P})$ and middle (M)

ultrasound contrast agents is still developing and experience with their most effective use is growing the exact role of ultrasound contrast agents in coronary artery examination remains to be established.

\section{Visualisation of the left anterior descending coronary artery}

With the patient in left decubitus position the transducer is placed at the left parasternal location and a parasternal short axis view of the great vessels is obtained. Slight change of the imaging plane so that it traverses the heart immediately below the pulmonary trunk allows for visualization of the left main (LM) and proximal LAD. These arteries may be relatively easily visualized in B-mode while Doppler examination is usually required to locate the more distal parts of the LAD (Fig. 6,7,8,9, movie 1, 2 [see Additional file 1, 2]). Depending on anatomical relations in a particular patient, sometimes it is easier to assess the proximal part of the LAD in a modified parasternal long axis views. The artery may be seen there as it traverses below the pulmonary trunk towards the interventricular groove (Fig. 10). The LM and proximal LAD may also be viewed using a modified apical five chamber view (traversing anterior wall of the heart) within the area lateral to the sinus of Valsalva (Fig. 11,12).

The middle and distal LAD may be assessed by placing the transducer in low parasternal position. The interventricular groove is located in short axis view and then the transducer is either tilted towards the base of the heart or rotated to obtain a modified long axis view (aligned parallel to the groove). Long axis view of the LAD can be obtained using either of these views (Fig. 13,14,15,16, movie 3 [see Additional file 3]). The middle and distal LAD can also be visualized using a modified apical 5chamber view (traversing anterior wall of the heart). The entire LAD together with some diagonal branches can sometimes be assessed in this view (Fig. 12). The apical window is often used for examination of distal LAD at the apex (Fig. 17,18) and even on the inferior wall of the ventricle (Fig. 19,20). Apical window is usually the best to obtain low Doppler angles for velocity measurements in the LAD. The LM and the full length of the LAD may also be visualized in apical 3-chamber view, although the motion artifacts, proximity of the lung and winding of the LAD along its way down to the apex make examination difficult.

The patient may then be placed in supine position. The transducer is moved to the subcostal location and a long axis view of the heart is obtained. Then the transducer is tilted cephalad aiming at the anterior wall of the left ventricle where long segments of the LAD can be visualized. However, the quality of LAD images obtained in this view is usually low and Doppler angle is suboptimal (Fig. $21,22)$.

\section{Visualisation of the left circumflex coronary artery}

Since it may be assumed, that the circumflex coronary artery leaves the coronary sulcus at its lowermost point (as can be seen on parasternal short axis view), the left part of the sulcus between aorta and lowermost point of the sulcus may be divided into two sections of approximately the same length, and segments of the circumflex artery within respective sections of the sulcus may be considered proximal and middle Cx (Fig. 3,4).

The Cx is visualized in parasternal short axis view with imaging plane traversing the heart immediately below the pulmonary trunk (Fig. 23, movie 1, 2, 4 [see Additional file $1,2,4])$. Sometimes it is better seen when the probe is directed just below the left atrial appendage (Fig. 24). Usually a slight change of probe orientation is necessary to visualize the middle $\mathrm{Cx}$ which runs down the coronary sulcus at the border between the atrium and the ventricle (Fig. 25, 26, movie 5 [see Additional file 5]). It is best vis- 


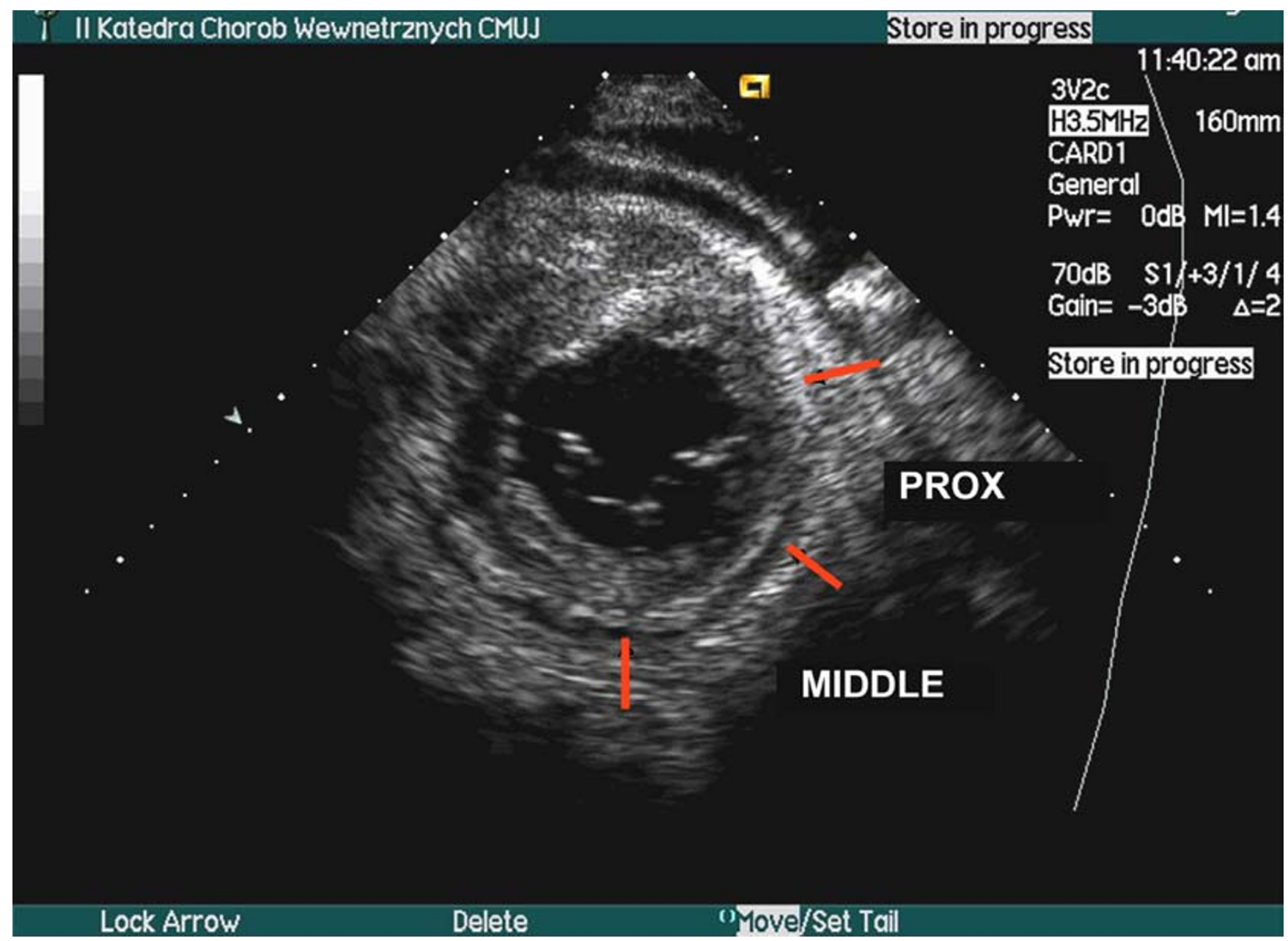

\section{Figure 4}

Parasternal short axis view at the level of mitral anulus. It is usually not possible to visualize proximal and middle part of the Cx in a single cross sectional view due to different spatial orientation of proximal and middle segments of the artery. Here, approximate location of proximal segment and location of middle segment in relation to the cross section of left ventricle at the level of mitral anulus is shown.

ualized with the imaging plane traversing the mitral annulus. Depending on anatomical relations in a particular patient, sometimes it is easier to assess the proximal and middle $\mathrm{Cx}$ in a modified parasternal long axis views. The proximal and middle Cx may be seen in this view within the lateral wall of the left ventricle (Fig. 10, 27, movie 6, [see Additional file 6]).

The transducer may then be moved to the apex and a modified five chamber view (traversing anterior wall of the heart) is obtained. Area lateral to the sinus of Valsalva is carefully searched and one may find there the Cx originating from the LM. A reliable measurement of the flow velocity within the initial part of the $\mathrm{Cx}$ may then be per- formed. Further segment of the Cx can also be visualized in B-mode, but as this part of $\mathrm{Cx}$ with this ultrasonic window runs almost perpendicular to the ultrasonic beam and is located several centimeters away from the transducer, this particular view is of limited use for the assessment of the Cx (Fig. 28).

We have never managed to visualize the distal $\mathrm{Cx}-$ at the infero-lateral wall of the ventricle.

\section{Visualisation of the right coronary artery}

The parasternal short axis plane may be used to visualize the proximal RCA (Fig. 29). It may be relatively easily visualized in B-mode (Fig. 30) while Doppler examination is 


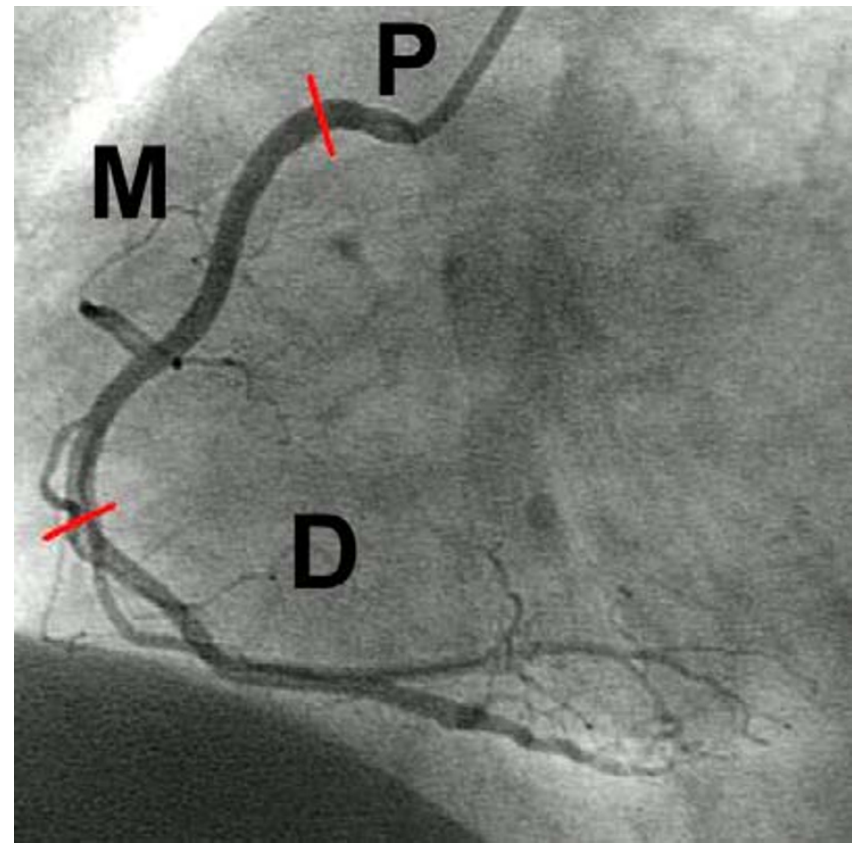

Figure 5

Angiography of the right coronary artery (RCA) in LAO projection. Division of the RCA into segments: proximal $(P)$, middle (M) and distal (D) - the posterior descending branch

usually required to locate its middle and distal parts. Depending on anatomical relations in a particular patient, sometimes it is easier to assess the proximal RCA in a modified parasternal long axis views. The proximal RCA may be seen in this view running along the anterior wall of the aorta (Fig. 31). The proximal RCA can infrequently be visualized using apical window, but this view allows for recording of the flow velocity spectrum (Fig. 32, 33).

The middle RCA is visualized with the patient in supine position. The transducer is placed in subcostal location and the short axis view of the heart is obtained. The anterior wall of right ventricle at the coronary sulcus is carefully scanned and it is where good quality middle RCA images at low Doppler angles can be obtained (Fig. 34,35,36, movie 7 [see Additional file 7]).

The distal RCA can be assessed using apical window. An apical four chamber view is obtained and then the transducer is tilted more perpendicular to the chest so that the imaging plane traverses the inferior wall of the heart and the posterior interventricular groove. Good quality images of the right posterior descending coronary artery usually distal part of the RCA - can be obtained in this way. Alternatively, a modified 2-chamber view (crossing the heart along the posterior interventricular groove) can be used (Fig. 37,38,39, movie 8 [see Additional file 8]).
Table I: Planes used for transthoracic examination of coronary arteries. Best results should be expected when views typed in bold are chosen

\begin{tabular}{|c|c|c|c|}
\hline LCA & LM/proximal LAD & Middle LAD & Distal LAD \\
\hline & mod. PSAX & mod. PSAX & AP3Ch \\
\hline & mod. PLAX & mod. PLAX & $\bmod$ AP5Ch \\
\hline & $\bmod \mathrm{AP} 5 \mathrm{Ch}$ & $\bmod$ A5PCh & \\
\hline & $\bmod \mathrm{AP} 3 \mathrm{Ch}$ & $\bmod A P 3 C h$ & \\
\hline & $\bmod$ SubLAX & $\bmod$ SubLAX & \\
\hline \multirow[t]{4}{*}{$C x$} & Proximal Cx & Middle Cx & Distal Cx \\
\hline & mod. PSAX & mod. PSAX & $?$ \\
\hline & mod. PLAX & mod. PLAX & \\
\hline & $\bmod \mathrm{AP} 5 \mathrm{Ch}$ & $\bmod \mathrm{AP} 5 \mathrm{Ch}$ & \\
\hline \multirow[t]{4}{*}{$\mathrm{RCA}$} & Proximal RCA & Middle RCA & Distal RCA (RPD)* \\
\hline & mod. PSAX & mod SubSAX & mod. AP4Ch \\
\hline & mod. PLAX & & $\bmod \mathrm{AP} 2 \mathrm{Ch}$ \\
\hline & $\bmod \mathrm{AP5Ch}$ & & $\bmod$ SubLAX \\
\hline
\end{tabular}

Abbreviations used in the table: LCA - left coronary artery LM - left main coronary artery LAD - left anterior descending coronary artery Cx - left circumflex coronary artery RCA - right coronary artery RPD* - right posterior descending (usually a distal branch of the RCA, but may arise from the $C x$ when the left coronary artery is dominant) mod - modified PSAX - parasternal short axis view PSLAX parasternal long axis view AP5Ch - apical 5-chamber view AP3Ch apical 3-chamber view AP2Ch - apical 2-chamber view SubSAX subcostal short axis view SubLAX - subcostal long axis view

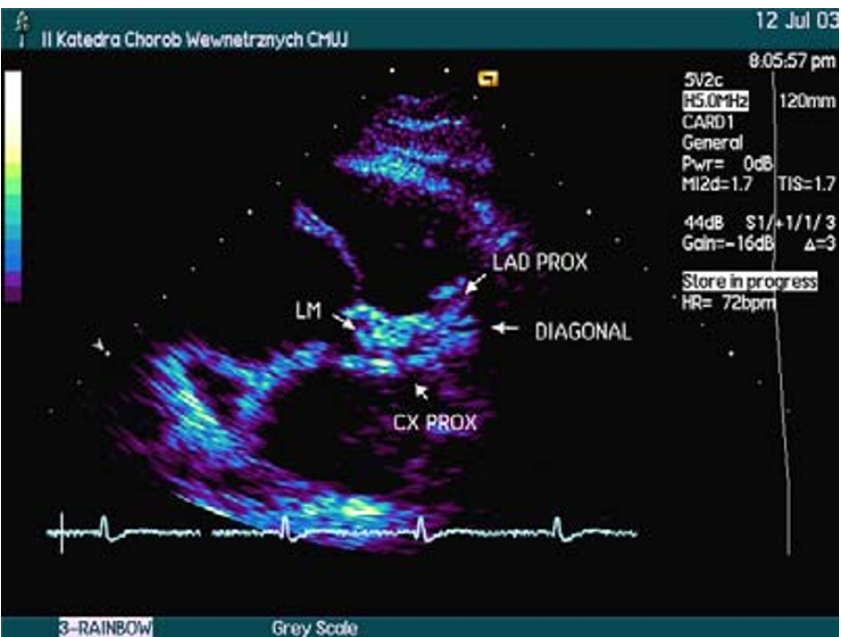

Figure 6

Proximal part of left coronary artery in B-mode, modified parasternal short axis view. LM - left main coronary artery. LAD PROX - proximal part of the left anterior descending coronary artery, Cx PROX - proximal part of the left circumflex coronary artery, DIAGONAL - proximal part of first diagonal artery 


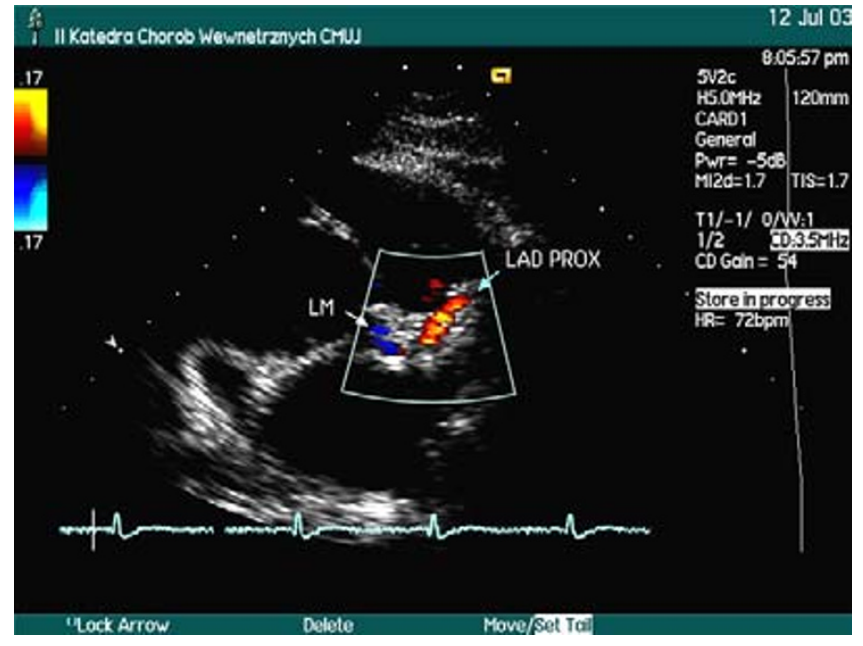

Figure 7

Proximal part of left coronary artery in color Doppler, modified parasternal short axis view. LM - left main coronary artery. LAD PROX - proximal part of the left anterior descending coronary artery

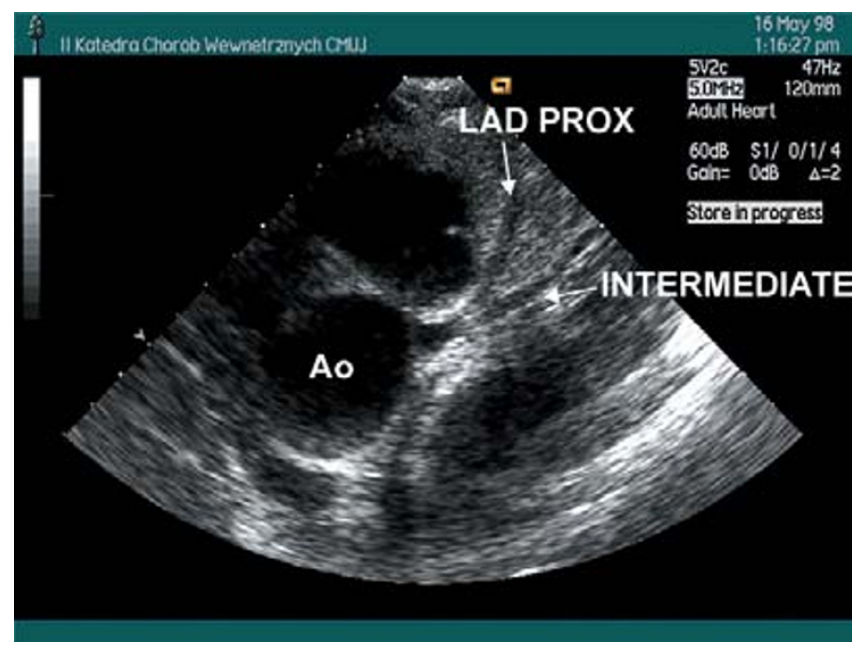

Figure 8

Proximal part of left coronary artery in B-mode, parasternal short axis view. LAD PROX - proximal part of the left anterior descending coronary artery, INTERMEDIATE - strong intermediate branch See movie I [see Additional file I]

Normal velocity spectrum and typical velocities of flow

Flow in coronary arteries is bi-phasic, predominantly diastolic. Therefore, the coronary artery flow velocity spectrum consists of two forward flow components: a lesser, dome-like, systolic, and a greater, diastolic (Fig. 40). The



Figure 9

Proximal part of left coronary artery in color Doppler, parasternal short axis view. See movie 2 [see Additional file 2]

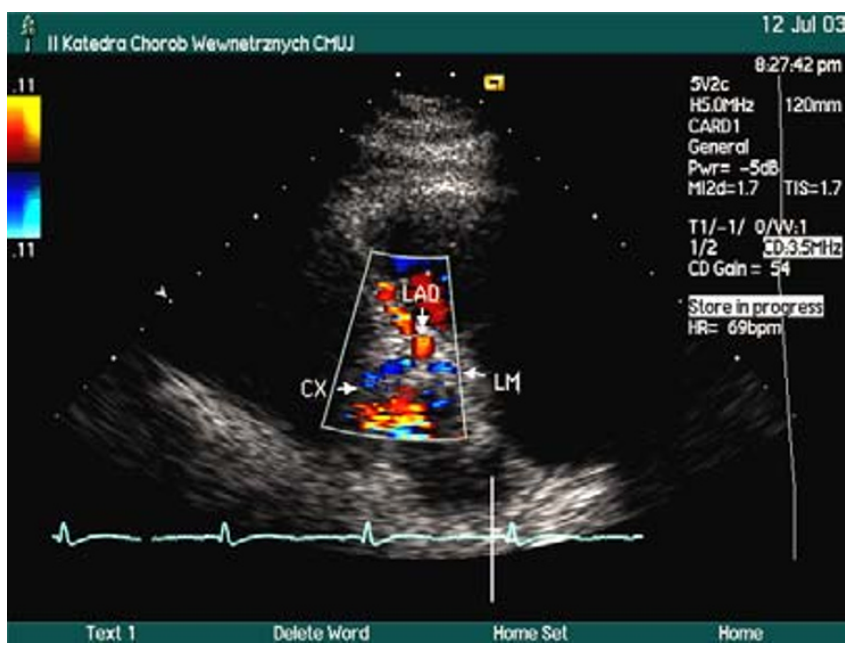

\section{Figure 10}

Proximal parts of the LAD and Cx in color Doppler; modified parasternal long axis view

direction and velocity of flow change in different pathologies. The forward systolic flow velocity decreases and there may even be a systolic flow reversal in the LAD in hypertrophic cardiomyopathy [18]. The proportion of maximal flow velocities in diastole to systole change distal 


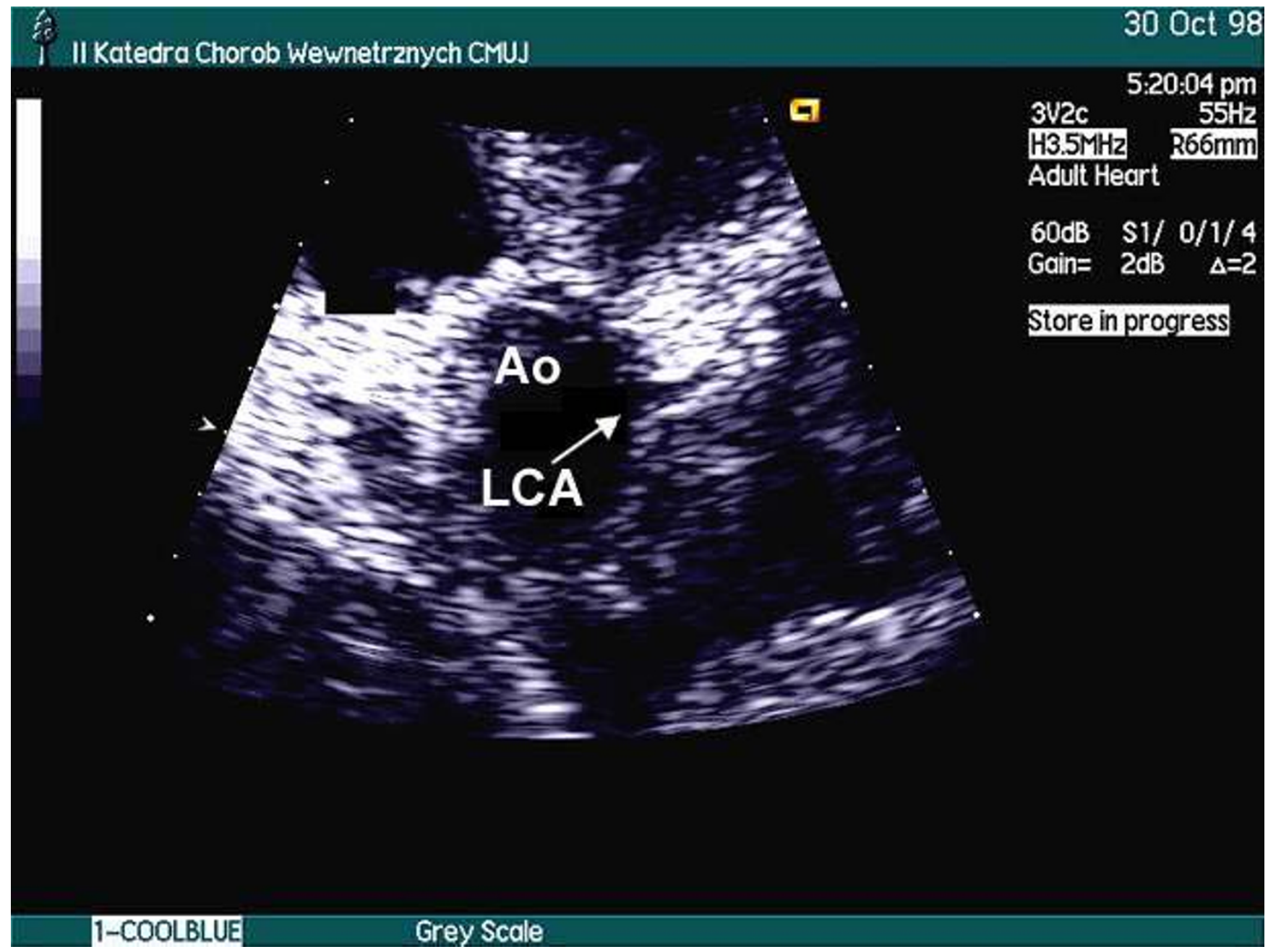

\section{Figure I I}

Left main coronary artery (LCA) arising from aorta (AO) in B-mode; modified apical 5-chamber view.

to a significant LAD stenosis. Flow velocities vary considerably depending on many factors: the heart rate, blood pressure, thickness of myocardium, coexistent heart disease, medications, perhaps even on vessel involvement in the formation of collateral circulation [19-21]. It increases at the site of stenosis. In a prospective study of sequential patients referred for coronary angiography we found maximal flow velocity within the LAD, Cx and RCA in the range of $0,7 \mathrm{~m} / \mathrm{s}$ for proximal parts of non-stenosed arteries and somewhat lesser values for their distal parts [10], but there are wide variations in the literature regarding maximal flow velocity in non-stenosed coronary arteries.

The spectrum recorded on transthoracic coronary artery examination is frequently devoid of a discernible systolic component, most probably due to movements of the heart (Fig. 41). For this reason the estimation of mean velocity and velocity time integral by TTE examination of coronary arteries may be impossible.

\section{Hints concerning machine settings}

There is a choice of ultrasound transducers available which may be used to examine the coronary arteries. The higher frequency transducers provide high resolution Bmode images and improved sensitivity of Doppler examination (both color and pulsed wave [PW] spectral Doppler). The high frequency transducers may be most successfully used when apical area is scanned - i.e. for examination of the distal LAD. The lower frequency transducers provide a good penetration and higher Nyquist limit for PW-spectral and color Doppler examination at the expense of lower resolution and lower 


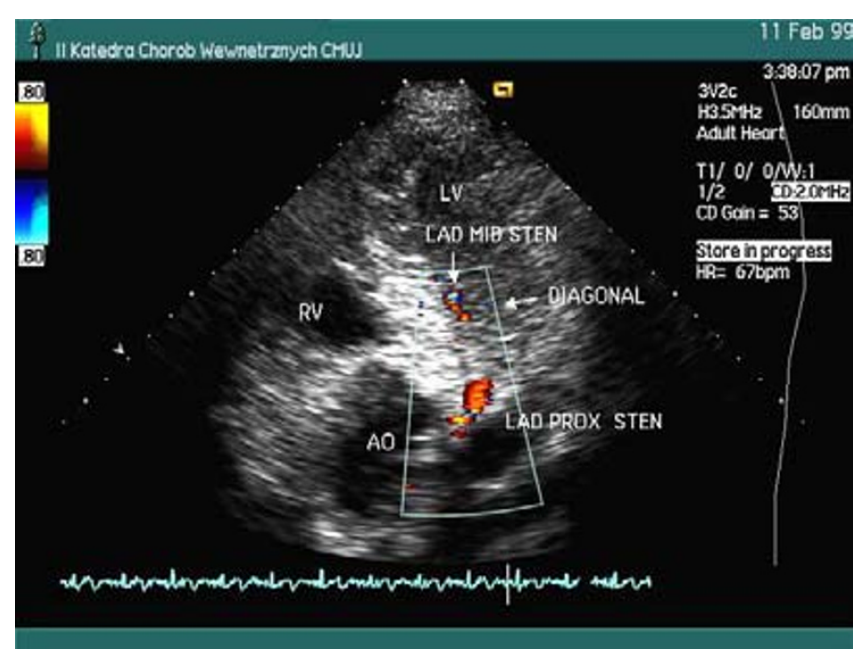

Figure 12

Left coronary artery in color Doppler; modified apical 5chamber view. Parts of the left main and LAD coronary arteries are seen. Color aliasing indicating local stenosis of proximal (LAD PROX STEN) and middle LAD (LAD MID STEN) are seen. Color aliasing was also noted in proximal part of a diagonal branch (not shown in this frame). Duplex criteria of significant stenosis were met for all stenoses, the diagnosis was subsequently confirmed by coronary angiography

sensitivity of Doppler exam. Nevertheless, they seem to be the optimal choice for scanning of RCA, Cx and proximal and middle LAD.

We suggest optimal quality B-mode image should be obtained before one starts to look for coronary arteries. When poor quality of B-mode image does not allow for visualization of coronary arteries one may decrease the dynamic range of $\mathrm{B}$-mode scanning, change the ultrasonic window or use the harmonic mode for B-mode, which we believe greatly facilitates scanning of the coronary arteries.

Then the color Doppler is turned on and the Nyquist limit for color examination is in most instances adjusted to 0,4 $-0,5 \mathrm{~m} / \mathrm{s}$. Too little the Nyquist limit the more color Doppler artifacts obscuring the images are encountered. On the other hand, too high a Nyquist will make detection of low velocity flow within the coronary arteries impossible. Gain is increased until large amount of color artifacts almost obliterate the image. It is then turned down a little and the area where the artery should be located is carefully scanned. Further gain and Nyquist limit adjustments are performed when flow signal within the artery is detected. The size of the area where color Doppler examination is carried out should be kept at minimum. Some ultrasound machine manufacturers suggest a special color map should be used for coronary artery examination - a map, which does not discriminate between velocities to and away from the transducer. We, however, believe a regular color map is perfectly sufficient to this aim. If the artery cannot be localized, change of ultrasonic window (change of the distance between the vessel and transducer, change of Doppler angle), further optimization of B-mode image or readjustment of color settings (gain, size of the area, where color examination is carried out or Nyquist limit) or change of transducer frequency should be considered. If these fail an ultrasound contrast agent may be tried.

Usually a small part of the artery can be seen at first. Then, by moving step-by-step up and down the course of the vessel the entire artery (or its long part) may be assessed (Fig. 42, 43, movie 9 [see Additional file 9]).

Once the vessel is located velocity spectrum is recorded. As there is no general agreement on which Doppler angle is optimal for PW spectral Doppler (duplex) examination it is universally accepted that for the duplex scanning the angle should never exceed 60 degrees. The lower the angle the less the possible error introduced by any inaccuracies of Doppler angle estimation but at the possible price of sensitivity of recording of higher velocities. The latter may be particularly important in very tight stenoses where Doppler signal intensity may not be strong enough to allow for correct measurement of maximal velocity. In such cases it may be advisable to change the window so that the Doppler angle increases towards (but does not exceed) 60 degrees.

We usually use a $5 \mathrm{~mm}$ gate for pulsed Doppler (to narrow a gate would decrease the signal intensity and too wide would make the signal more "noisy"). Smaller gate (3 $\mathrm{mm}$ ) may be used when distal segment of the LAD is examined. We generally use the manufacturer default Doppler filter setting (as for regular echocardiographic examination).

\section{Artifacts of scanning. Sources of diagnostic errors}

Artifacts pose an important problem which make the examination exceptionally difficult, especially when the Cx or RCA are scanned. Most of them are caused by movements of the heart.

Often the flow of physiological amount of fluid within the pericardial sac generates a strong signal which may be confused with flow within a coronary artery (Fig. $43,44,45,46,47$, movie 10 [see Additional file 10]). The flow within the pericardial sac is most pronounced in systole, while the coronary artery flow is predominantly diastolic and this should allow for correct interpretation of images. 


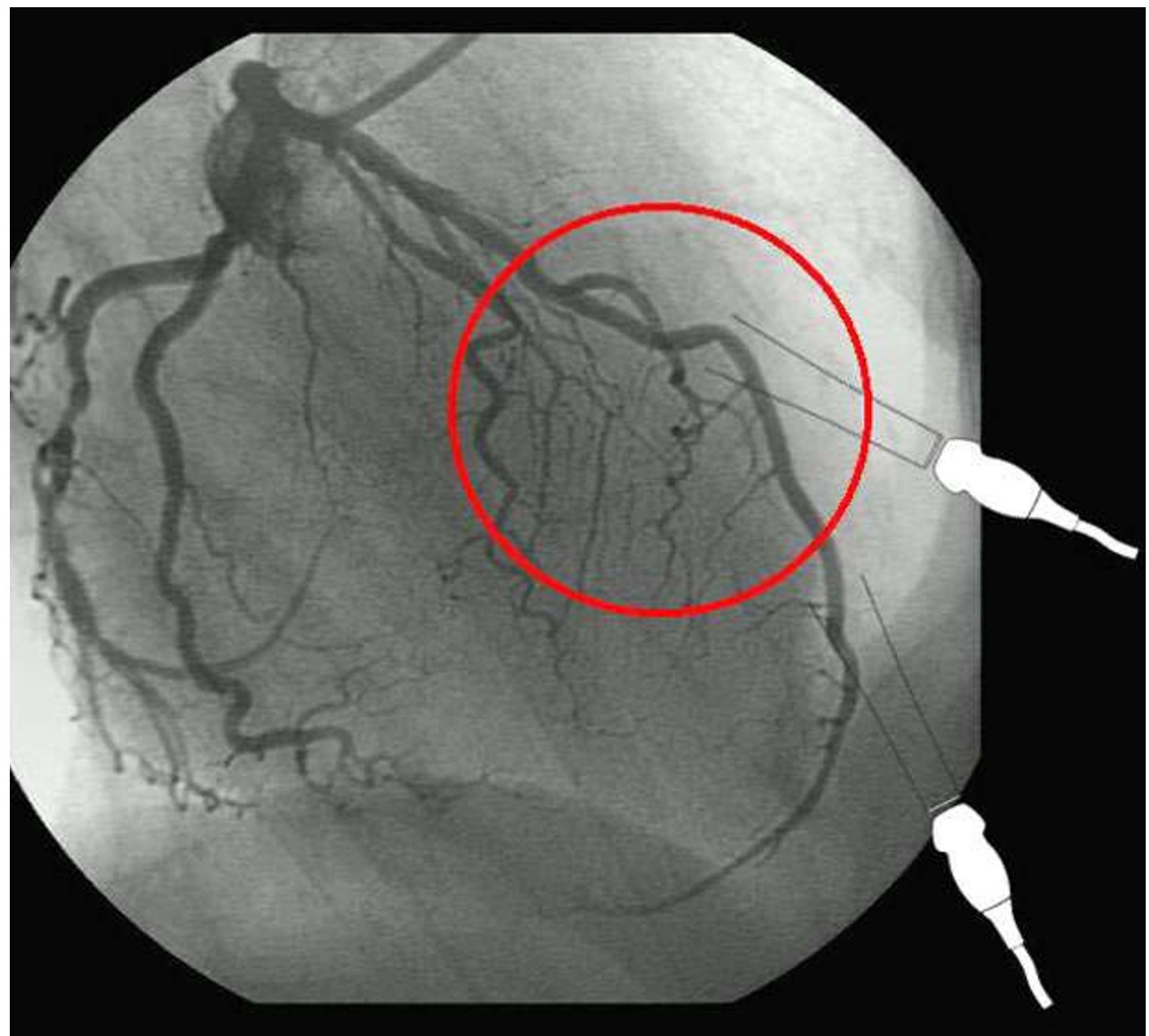

Figure 13

Angiography of the left coronary artery in RAO projection with caudal angulation. Middle segment of the LAD is marked by red circle for correlation with echocardiographic image

We have on a few occasions observed a characteristic artifact mimicking a very high velocity flow within the middle or distal LAD, which can be recorded both in color and spectral Doppler (Fig. 48,49, movie 11 [see Additional file 11]). When carefully examining the velocity spectrum one may notice then, that: 1 . the signal is not confined to diastole - it is rather recorded in a random way, without any definite relation to the cardiac cycle and 2 . the maximal velocity cannot be recorded due to aliasing.
When middle LAD is scanned some extracardiac vessels as well as a cardiac vein can be visualized on color Doppler examination, which may be confused with the LAD. However, spectral Doppler examination allows for proper distinction: the extracardiac arteries show a spectrum typical for peripheral arteries with predominantly systolic flow while veins show a steady, systolic-diastolic, low velocity flow which would cessate or significantly decrease on Valsalva maneuver. The left internal thoracic artery (LIMA) 


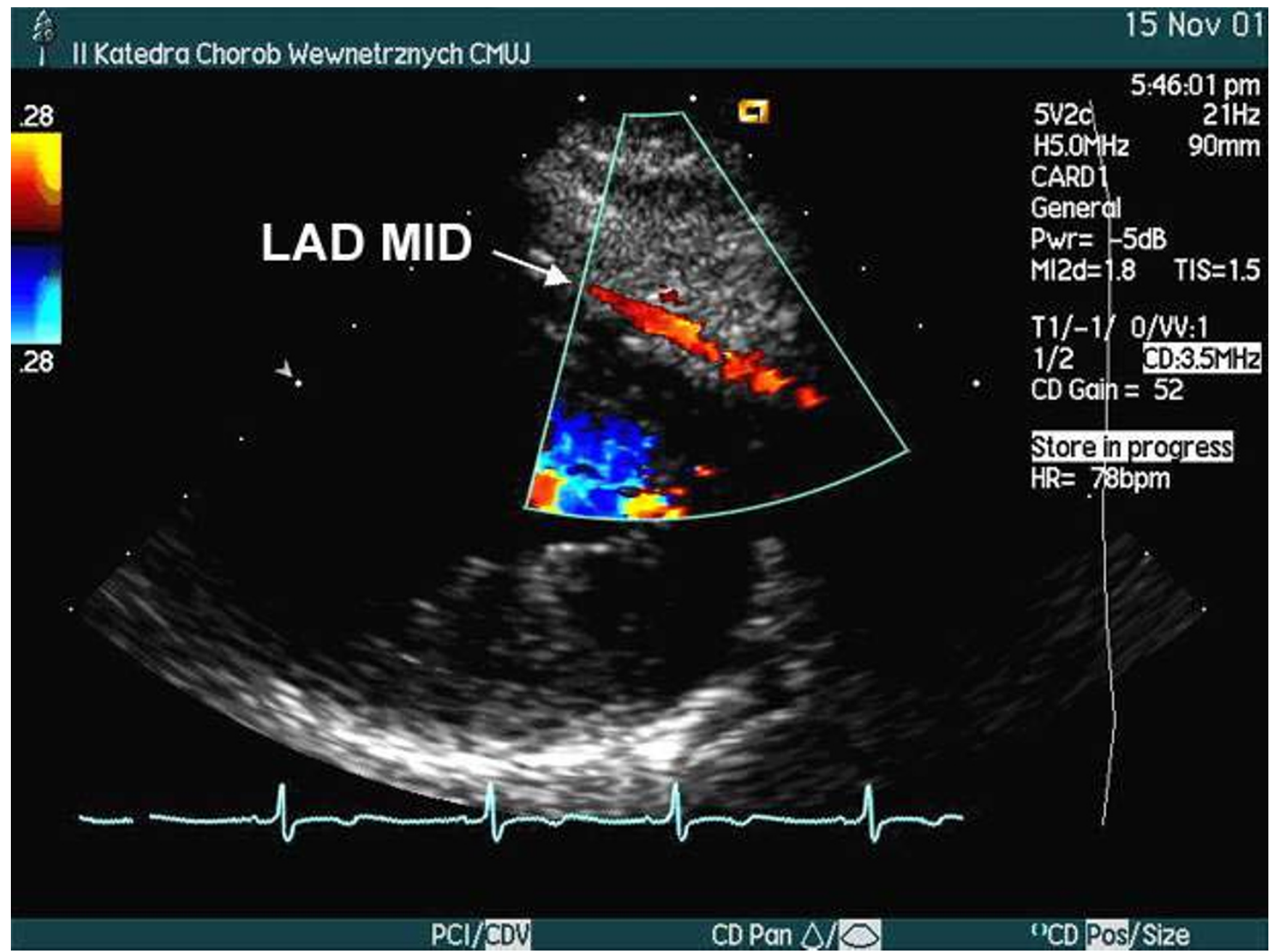

Figure 14

Middle LAD in color Doppler. Modified parasternal long axis view. See movie 3 [see Additional file 3]

may be confused with the LAD in cases, in which the LIMA was used for a coronary artery by-pass. The velocity spectrum within the LIMA has then a prominent diastolic component typical for the coronary arteries which may be misleading for a beginner. However, the systolic part of velocity spectrum recorded in LIMA is typical for a peripheral artery. Although the systolic component successively diminishes between the origin of the artery internal mammary artery and its anastomosis with the coronary artery, it is more pronounced, than the diastolic component and therefore easily discernible.

The posterior descending coronary artery which usually is a distal branch of the right coronary artery can arise from the Cx when left coronary artery is dominant, which may cause diagnostic mistakes.
Some large branches of coronary arteries - the intermediate coronary artery (Fig. 8, movie 1 [see Additional file 1]), diagonal and marginal branches can sometimes be visualized and confused with principal coronary arteries, most notably the LAD and Cx. It is especially likely when the principal artery is occluded and a branch enlarges and takes over its function. We have occasionally encountered this problem with regard to proximal LAD and proximal $\mathrm{Cx}$. It is easier to properly locate the LAD at its middle part, as it is clearly localized in the anterior interventricular groove. By moving step-by-step towards aorta or downwards to the apex without loosing the LAD from sight one may correctly identify the proximal and distal LAD. The fact, that distal LAD is the only artery which is visualized from a strictly apical transducer posi- 


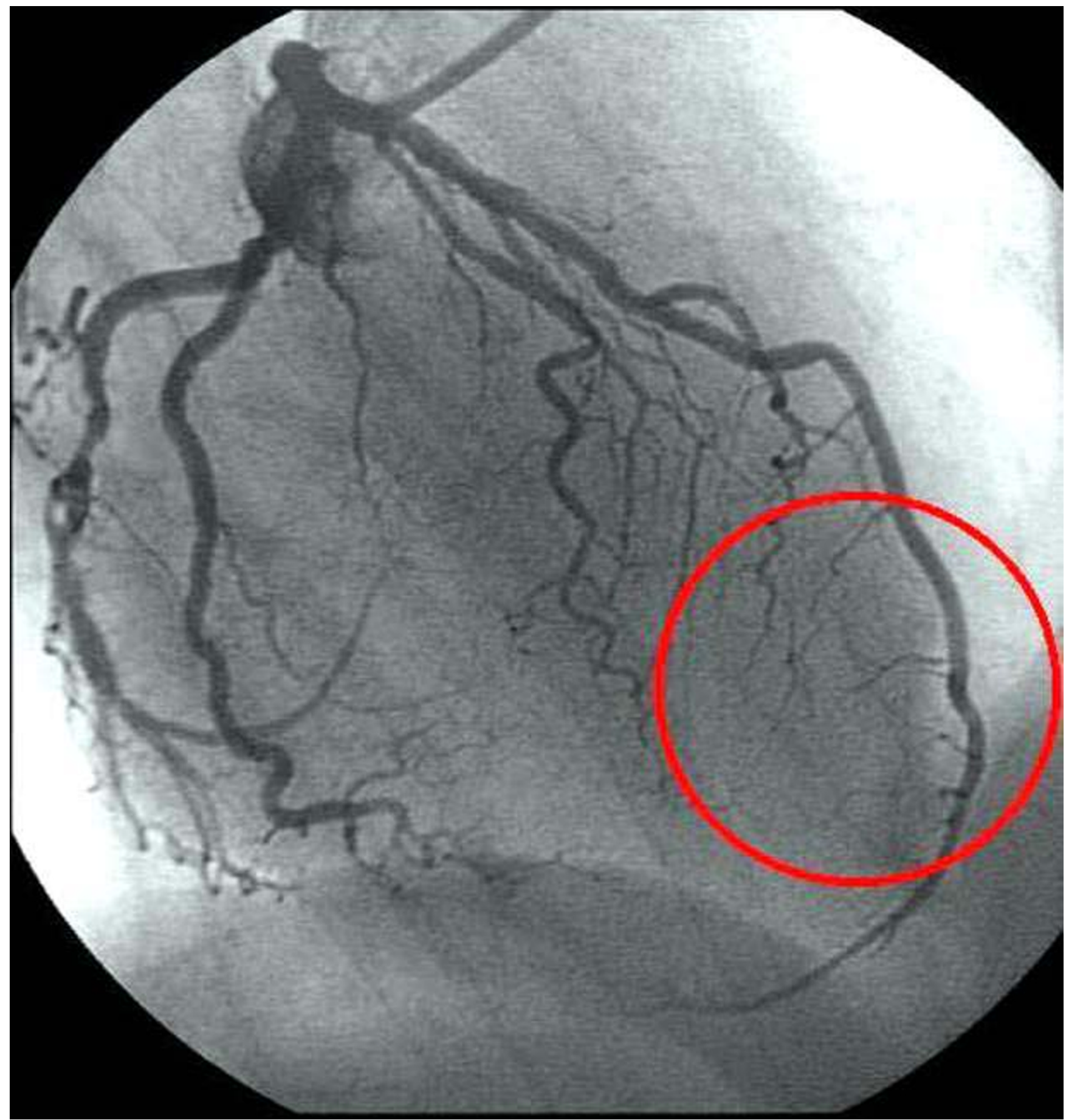

Figure 15

Angiography of the left coronary artery in RAO projection with caudal angulation. Distal segment of the LAD at the anterior wall of the ventricle is marked by red circle for correlation with echocardiographic image

tion and that it "winds" to the inferior wall of the heart is another clue (Fig. 20).
The differentiation between various vessels seen at the lateral and inferior wall of the ventricle may be challenging. 


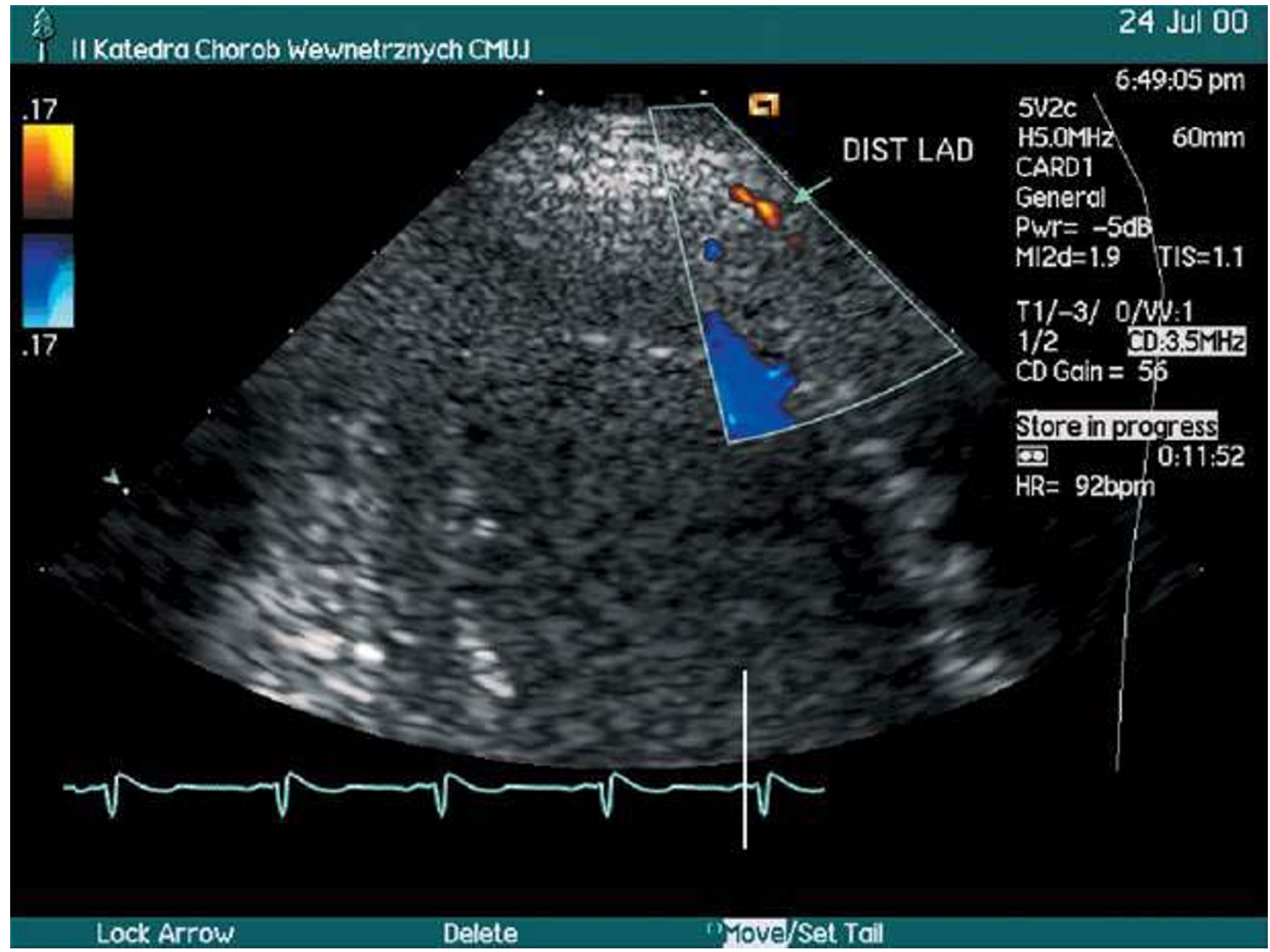

Figure 16

Distal LAD at the anterior wall of the left ventricle in color Doppler. Modified low parasternal long axis view

Improper machine settings, most notably choosing to high or too low Nuquist limit for color Doppler may result in failure to visualize the coronary arteries.

\section{Discussion: Practical aspects of TTE examination of coronary arteries}

Color duplex ultrasound examination is an invaluable tool for assessment of peripheral arteries. While studies are needed to determine if the TTE examination of long segments of coronary arteries will become a useful ancillary test in cardiology we can summarize our 5-year experience during which we have performed some 800 scans of the LAD, the $\mathrm{Cx}$ and/or the RCA as follows $[10,11]$ :
1. The examination is technically demanding, but the feasibility of visualization of the LAD is high and moderate with regard to the Cx and RCA [[9-11,22], table 2].

2. Although there are no firmly established Doppler criteria of coronary artery stenosis we have been quite successful using the criteria valid for peripheral arteries i.e. when at least doubling of maximal flow velocity within stenosis with regard to maximal flow velocity immediately proximal to stenosis was taken as a sign of $>50 \%$ reduction of vessel diameter. Similar results were obtained by Hozumi et al. who used a ratio of mean velocities of 2.22 combined with local color aliasing as a sign of restenosis after percutaneous coronary intervention within the LAD [22]. Retrospective analysis of our results has shown, that a local maximal flow velocity $>1.5 \mathrm{~m} / \mathrm{s}$ is also a reasonably 


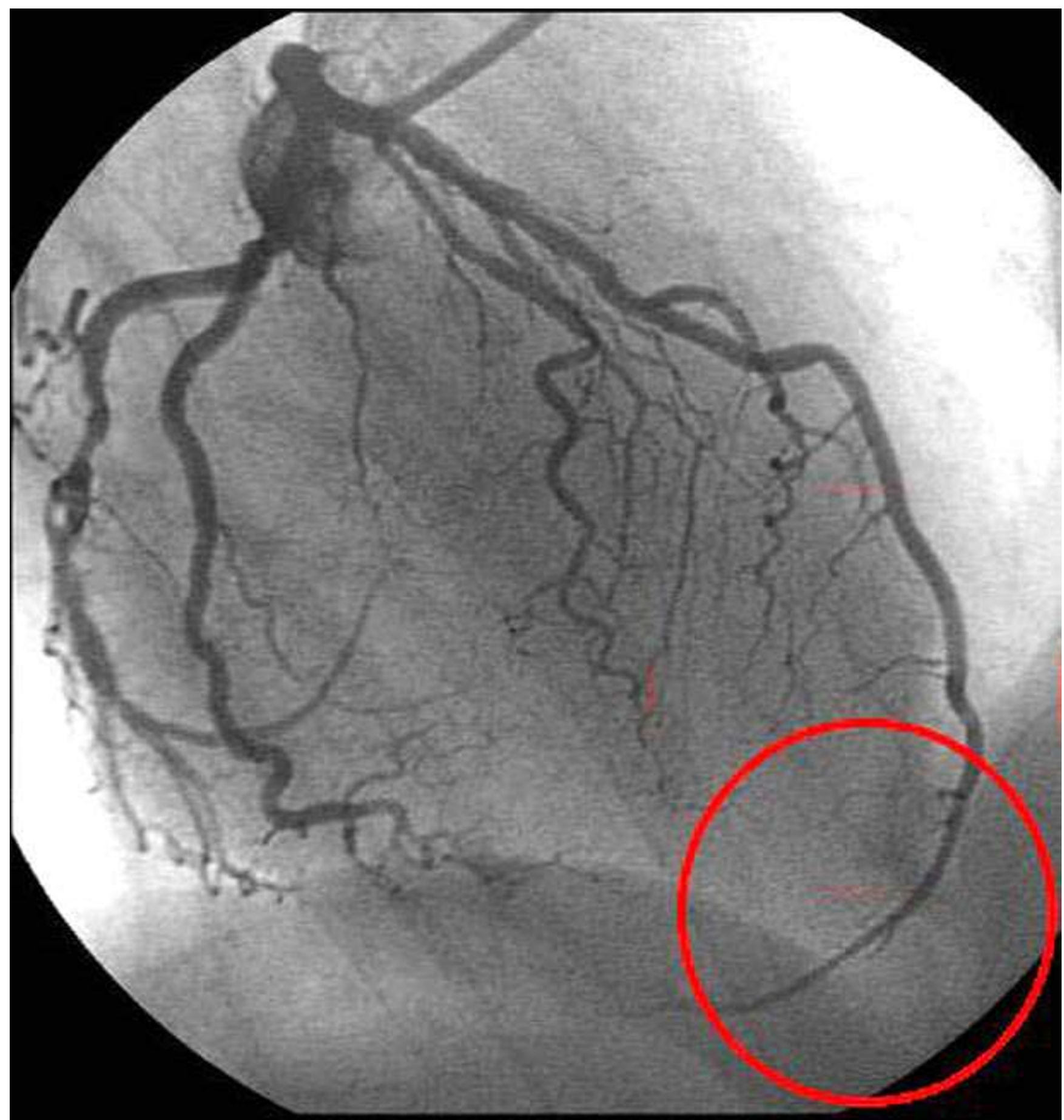

Figure 17

Angiography of the left coronary artery in RAO projection with caudal angulation. Distal segment of the LAD at the apex is marked by red circle for correlation with echocardiographic image

accurate sign of stenosis. The velocity ratio criterion seems more reliable than the criterion of maximal flow velocity, but the former may not be used in ostial lesions as there is no reference flow velocity for comparisons.
3. Once the artery can be visualized and spectral Doppler examination can be performed the accuracy of assessment (stenosis present / absent) is high. While our results await independent confirmation, we have shown that direct, 


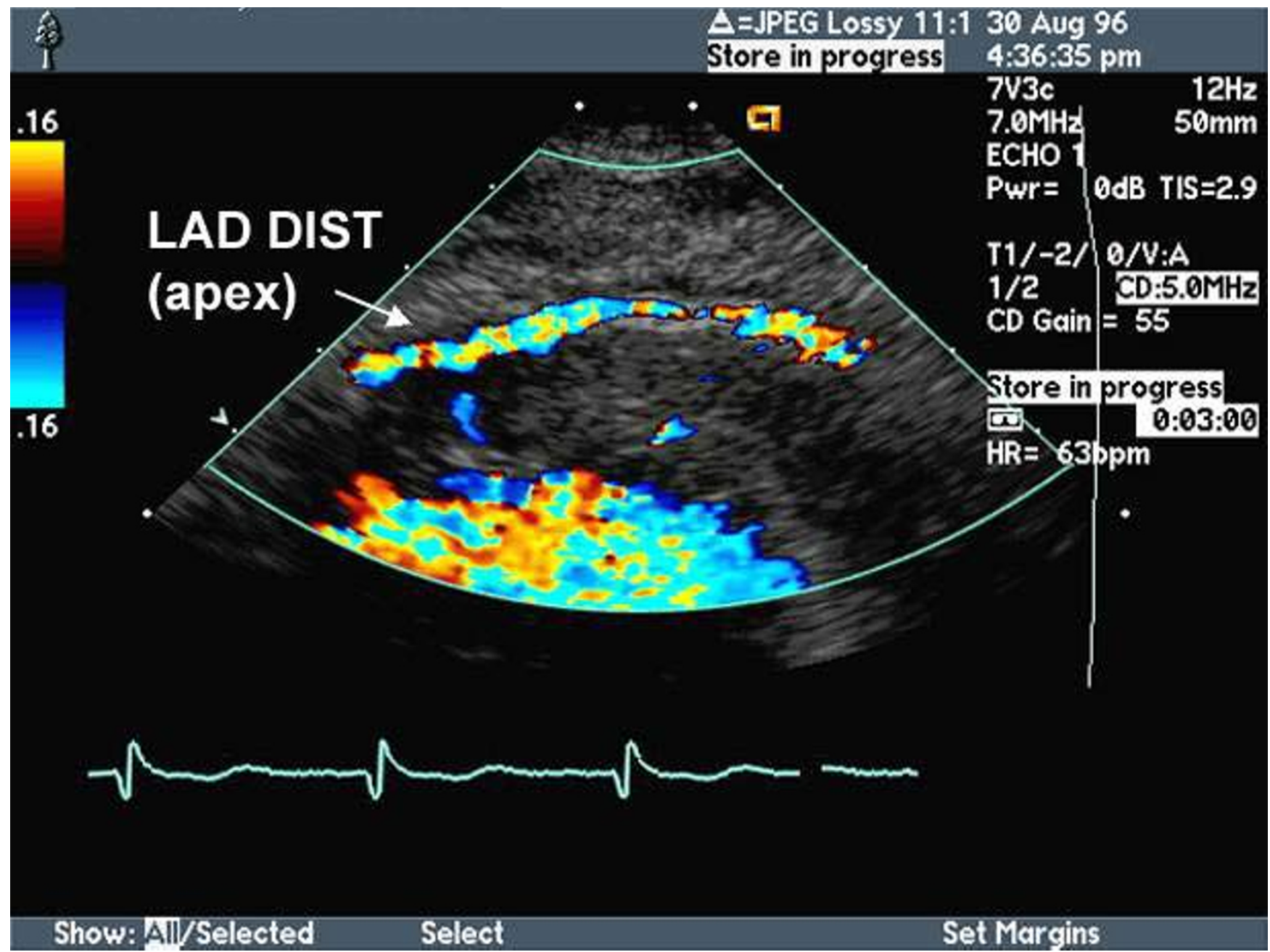

Figure 18

Distal LAD at the apex in color Doppler using a $7 \mathrm{MHz}$ transducer. Apical 3-chamber view. Courtesy of Acuson Corp.

transthoracic color Doppler examination of coronary arteries can be used to monitor restenosis after PCI within the LAD, Cx or RCA (Table 3).

4. It helps to know the anatomical details of coronary arteries (i.e. to review the coronary angiography) before the TTE of coronary arteries is performed. It can be easily done if TTE is used to monitor restenosis as films performed during the PCI are readily available. Our present results [11] are better than our previous [10], which may be explained by a learning curve, but also by the availability of coronary angiograms to the echocardiographer at the time of scanning. Most diagnostic errors relate to improper identification of principal coronary arteries i.e. taking a strong branch - septal or diagonal for the LAD and marginal for the $\mathrm{Cx}$.
5. Occasionally the diagnosis of coronary artery stenosis is so easy and straightforward that it can be made during a regular echocardiographic examination, taking only a few minutes to complete and not causing any meaningful prolongation of scanning (Fig. 50,51,52,53,54,55, movie 12, 13 [see Additional file 12, 13]).

\section{Authors' contributions}

$\mathrm{MK}$ and WB carried out the echocardiographic examinations prepared the pictorial materials and drafted the manuscript.

PPD participated in some of the studies and reviewed the manuscript.

All authors read and approved the final manuscript 


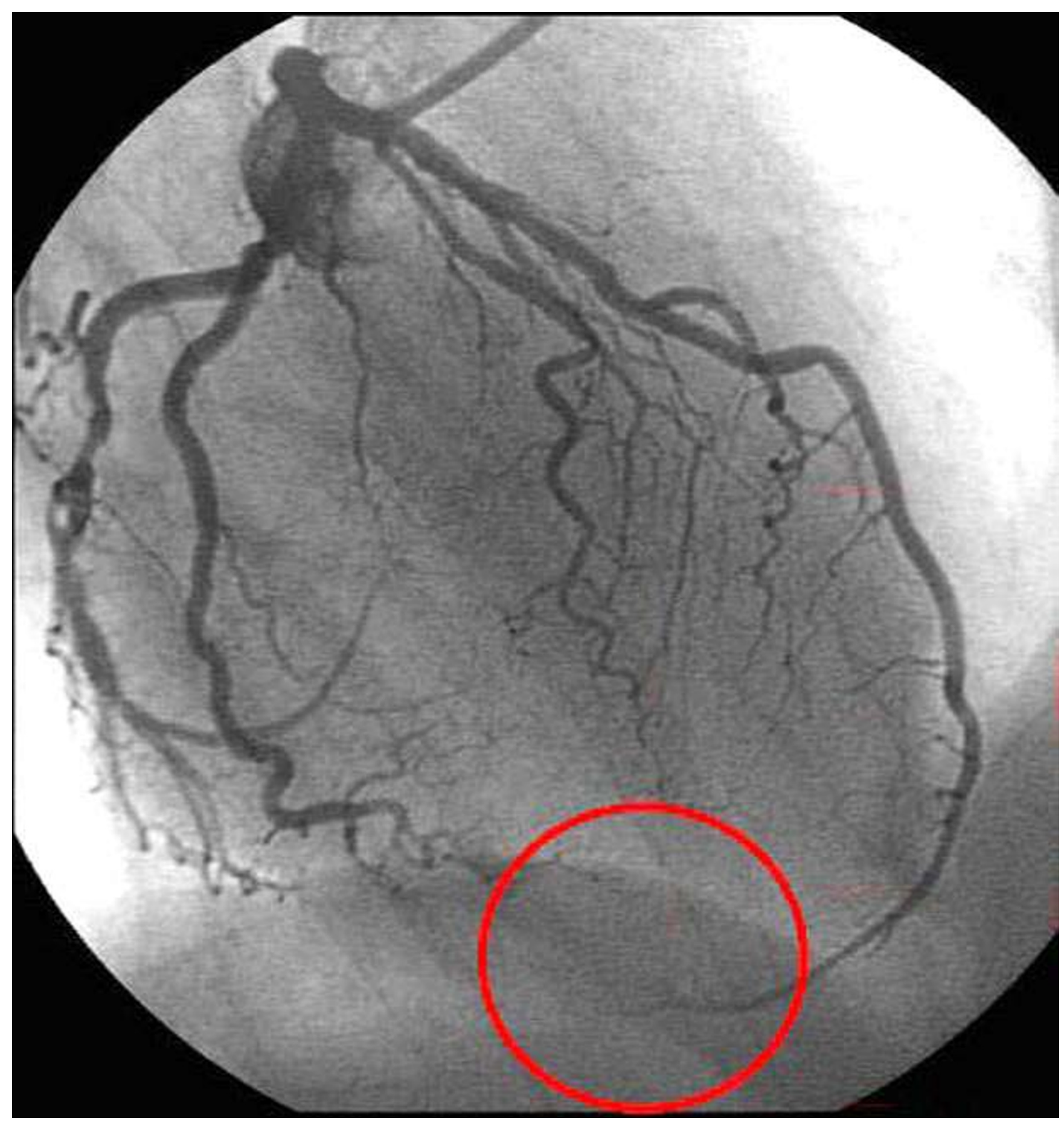

Figure 19

Angiography of the left coronary artery in RAO projection with caudal angulation. Distal segment of the LAD at the inferior wall of the heart is marked by red circle for correlation with echocardiographic image 


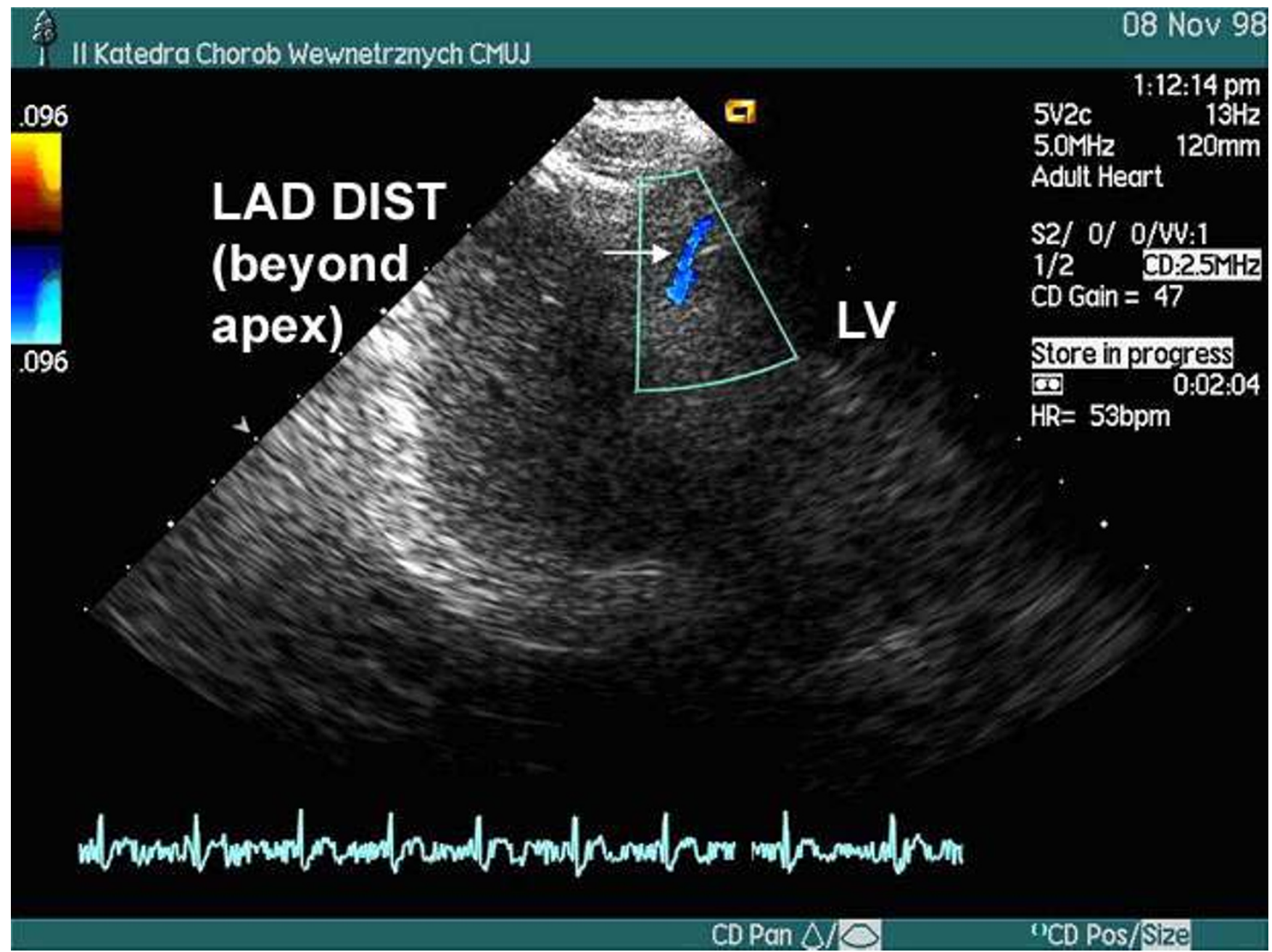

\section{Figure 20}

Distal LAD at the inferior wall of the heart in color Doppler. Apical 3-chamber view. Blood flow is depicted in blue. 




\section{Figure 2 I}

Left anterior descending coronary artery in color Doppler, modified subcostal long axis view. Ao - aorta, RV - right ventricle 


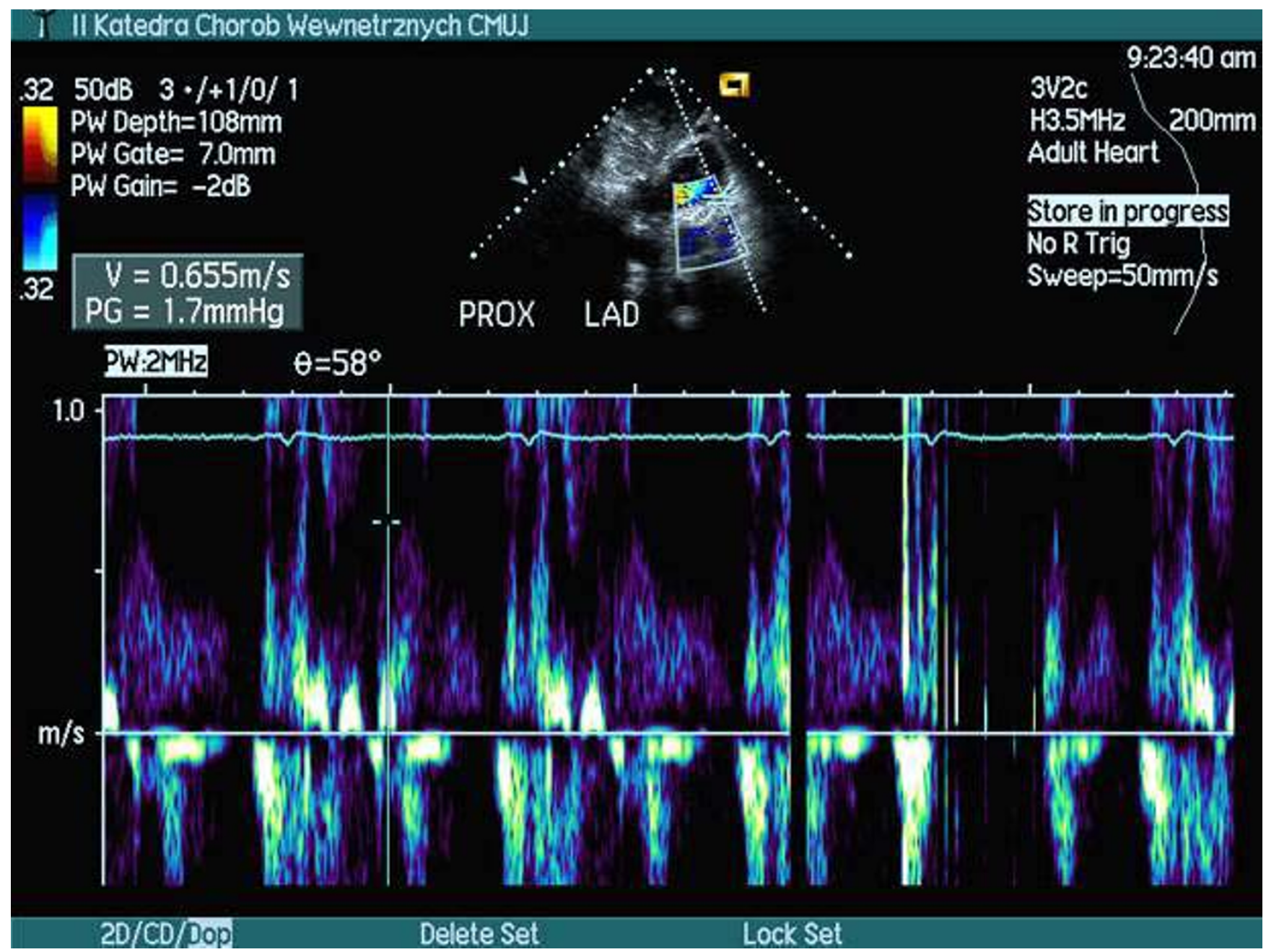

Figure 22

Duplex examination of the LAD in modified subcostal long axis view. 


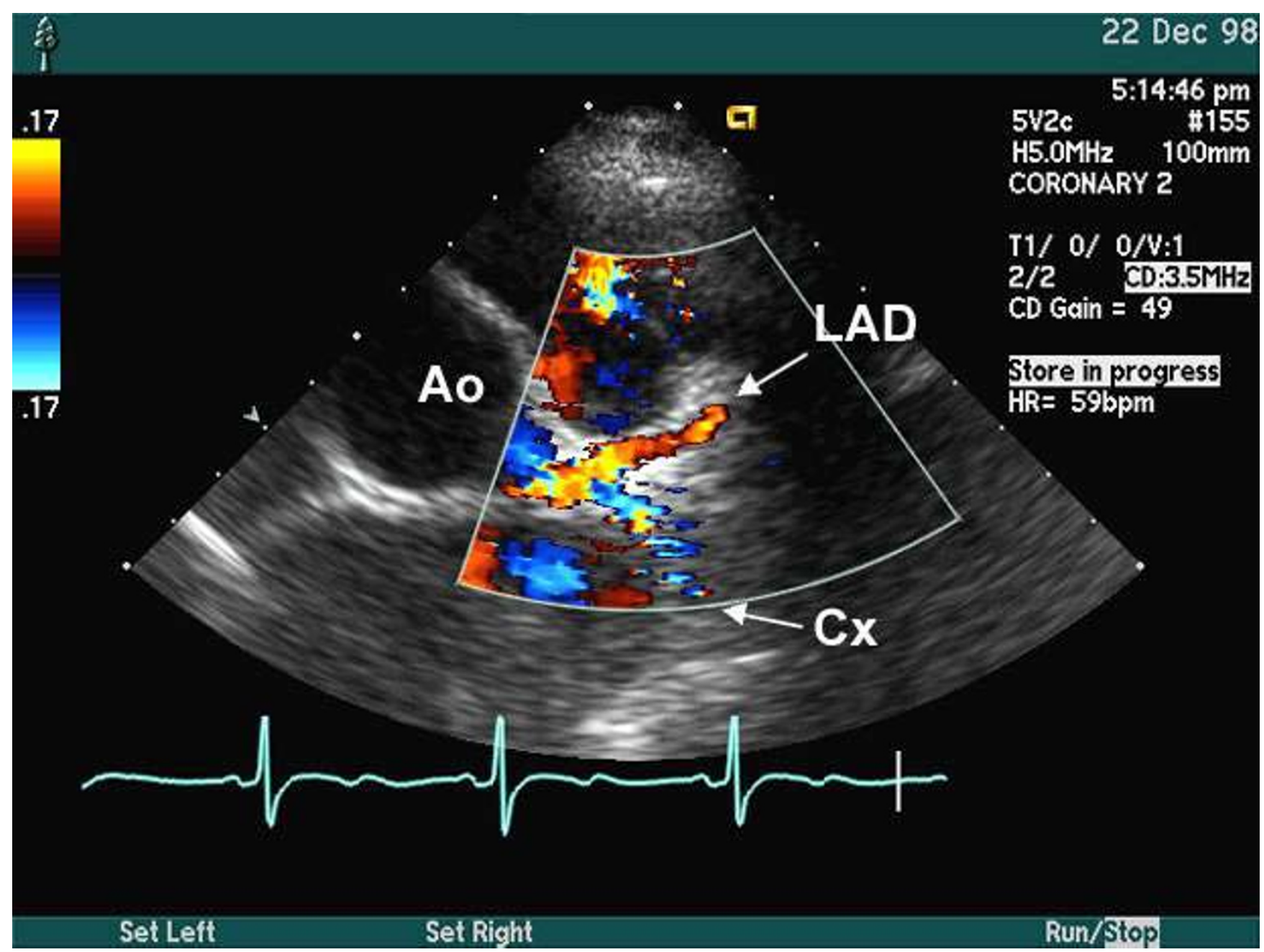

\section{Figure 23}

Proximal left circumflex, left main, proximal left anterior descending coronary arteries in color Doppler, prarasternal short axis view. Courtesy of Acuson Corp. See movie 4 [see Additional file 4] 


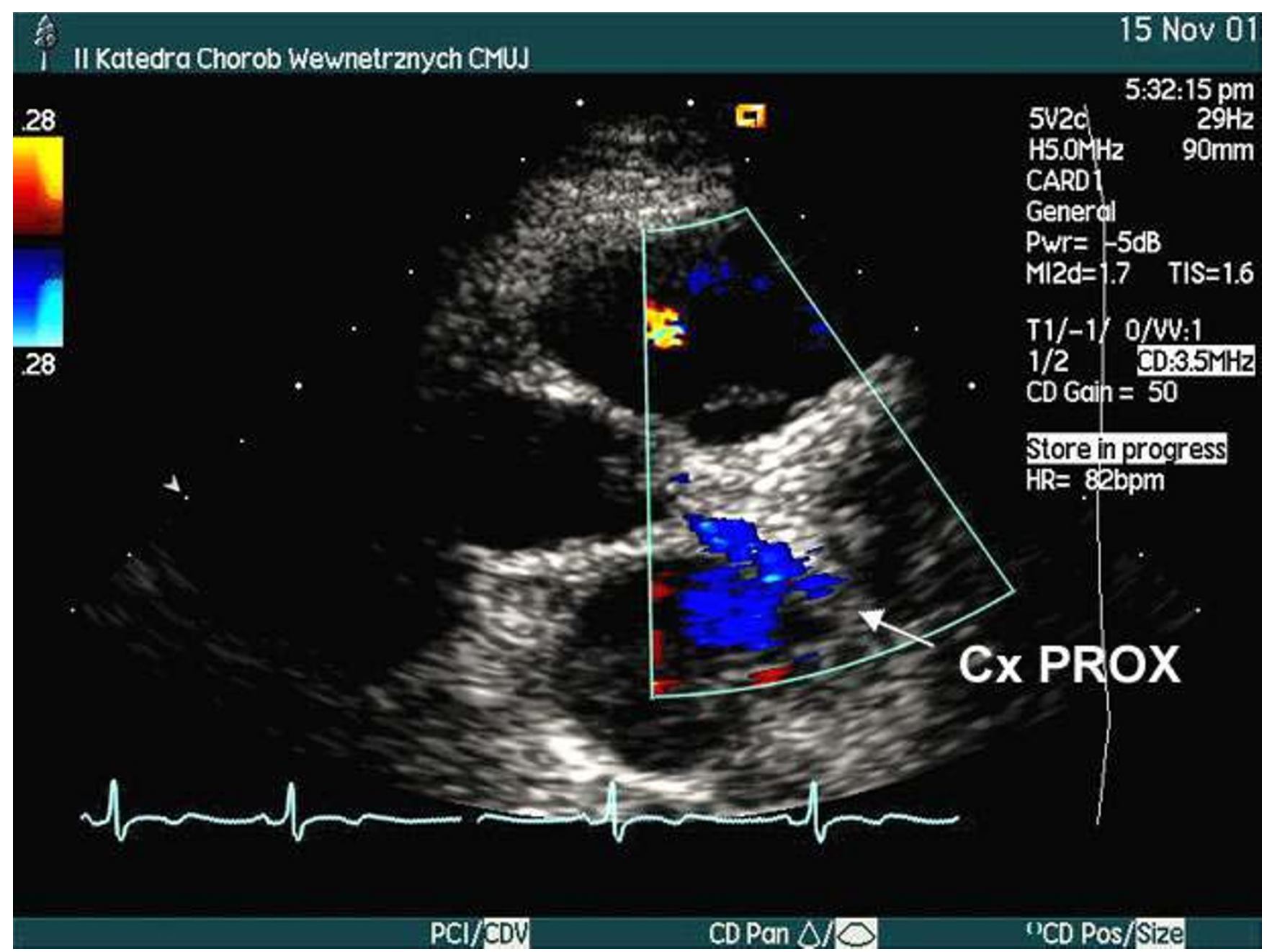

\section{Figure 24}

Proximal part of left circumflex coronary artery (Cx PROX) in color Doppler. Parasternal short axis view with imaging plane oriented just below the left auricle. 


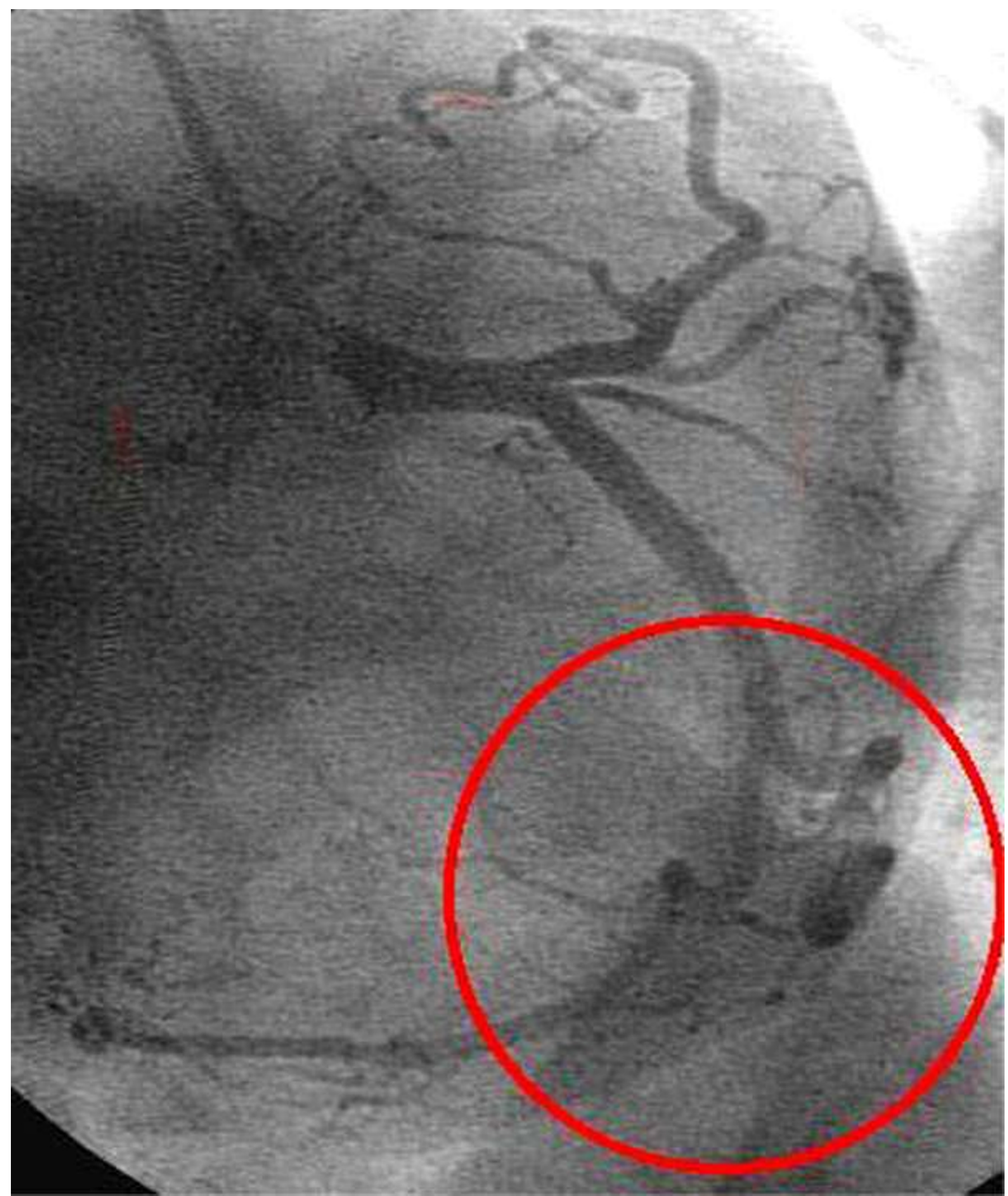

Figure 25

Angiography of the left coronary artery in LAO projection with caudal angulation. Middle segment of the Cx is marked by red circle for correlation with echocardiographic image 


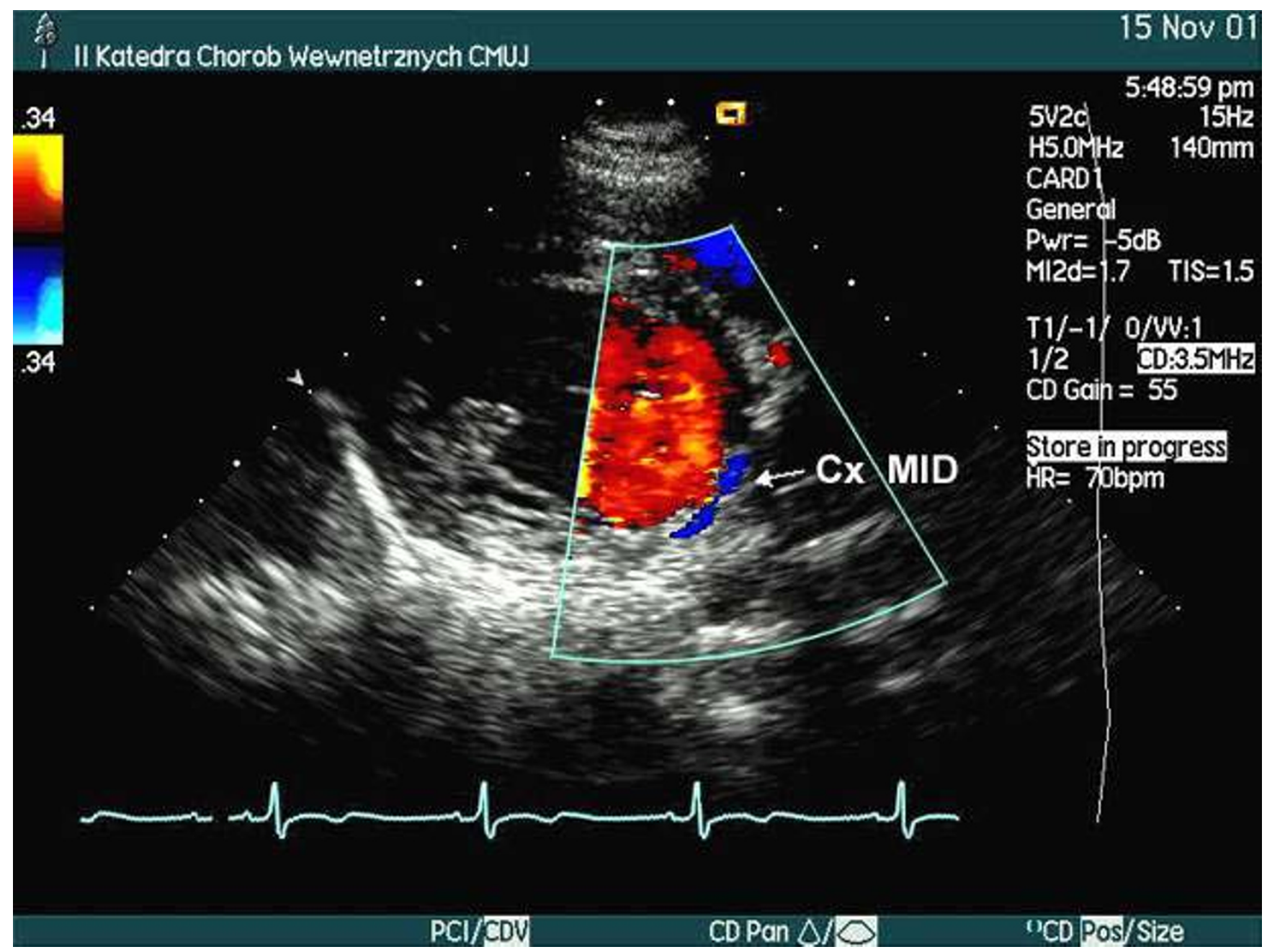

\section{Figure 26}

Middle part of left circumflex coronary artery in color Doppler. Parasternal short axis view, imaging plane traversing coronary sulcus. The flow in artery is depicted in blue. See movie 5 [see Additional file 5] 


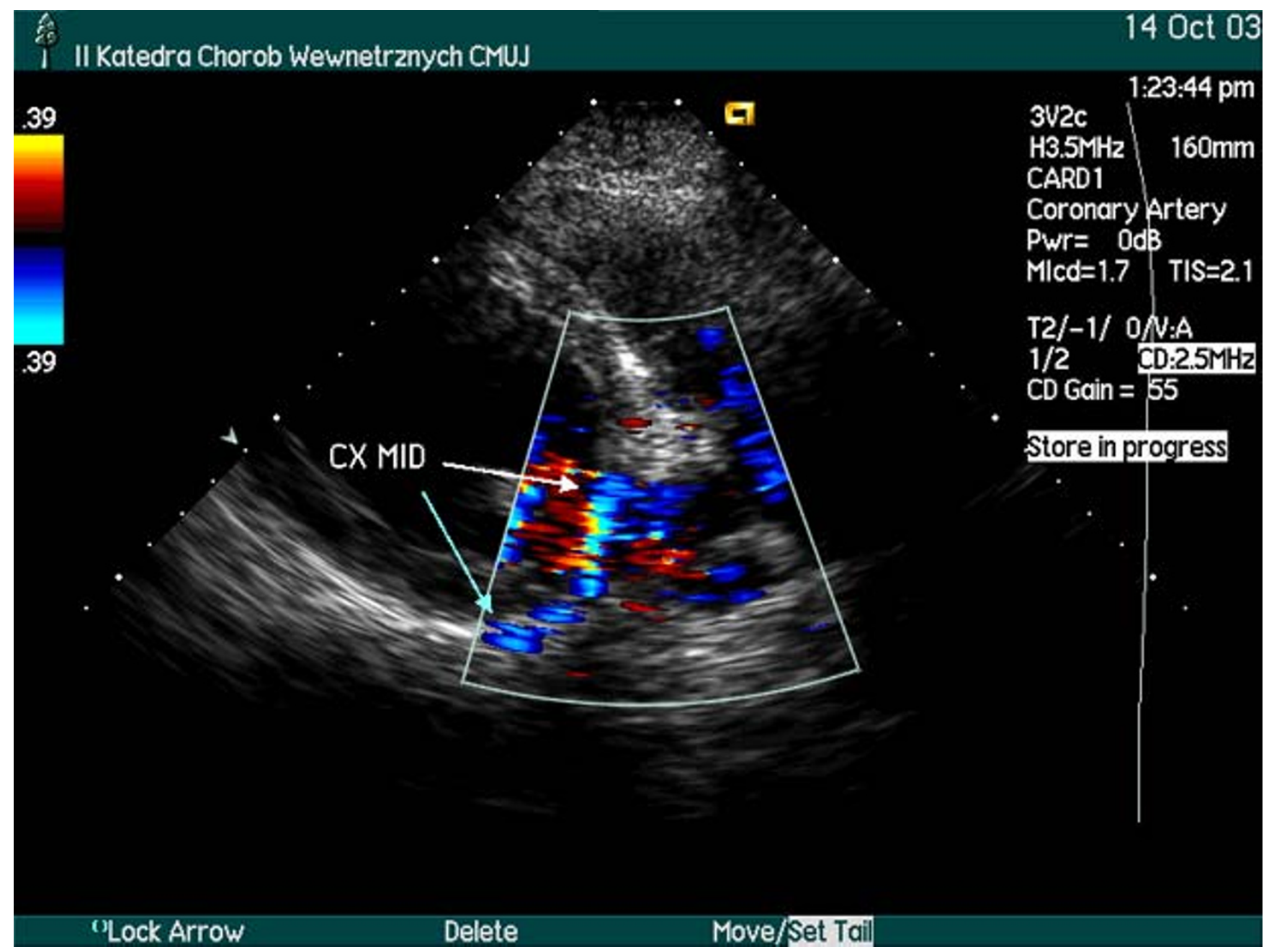

\section{Figure 27}

Middle part of left circumflex coronary artery in color Doppler. Modified parasternal long axis view, imaging plane traversing lateral wall of the left ventricle. The flow in artery is depicted in blue. See movie 6 [see Additional file 6] 


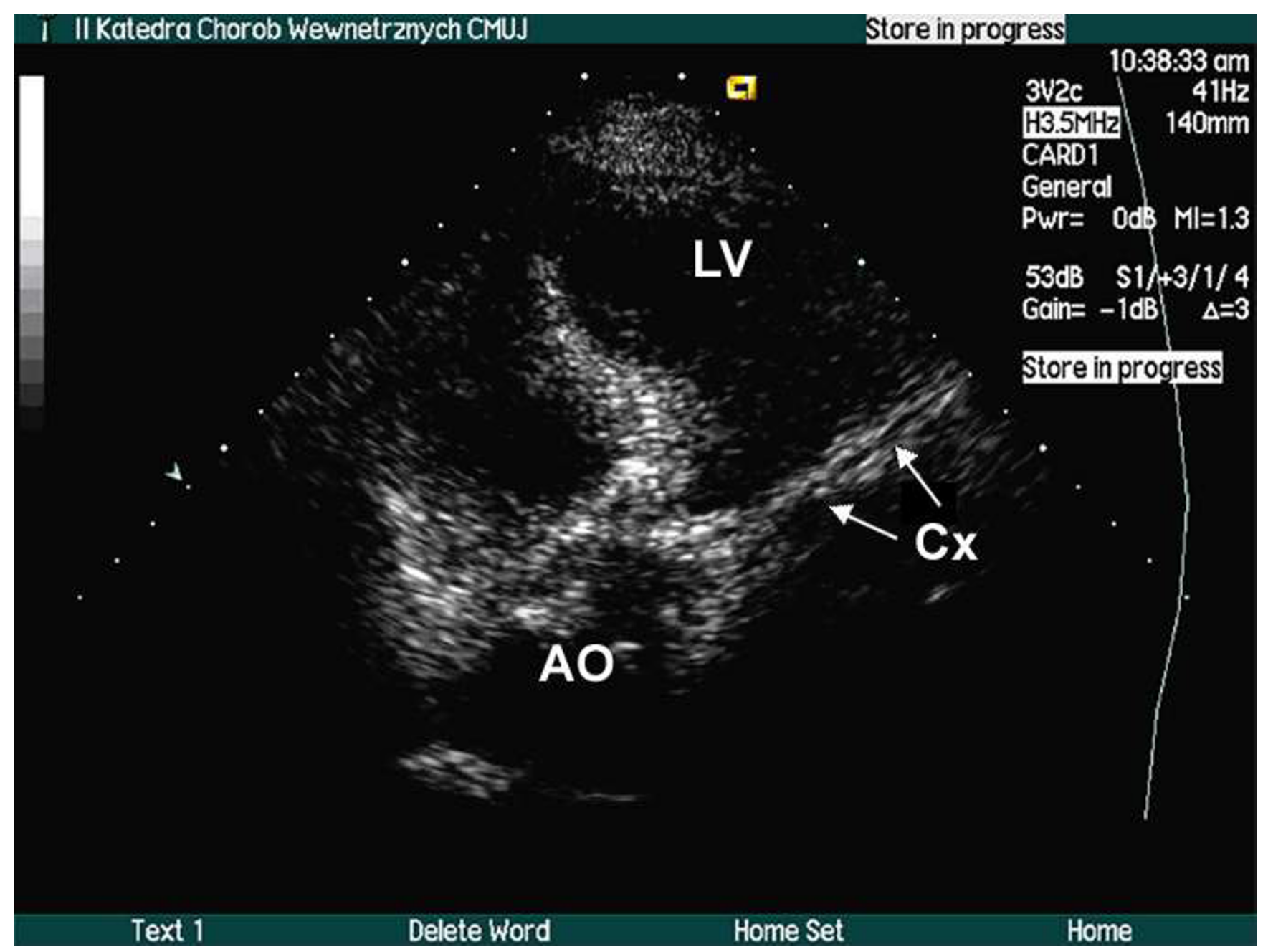

Figure 28

Proximal part of left circumflex (Cx) coronary artery in B-mode, modified apical 5-chamber view. 




Figure 29

Proximal part of righ coronary artery in color Doppler, parasternal short axis view. 


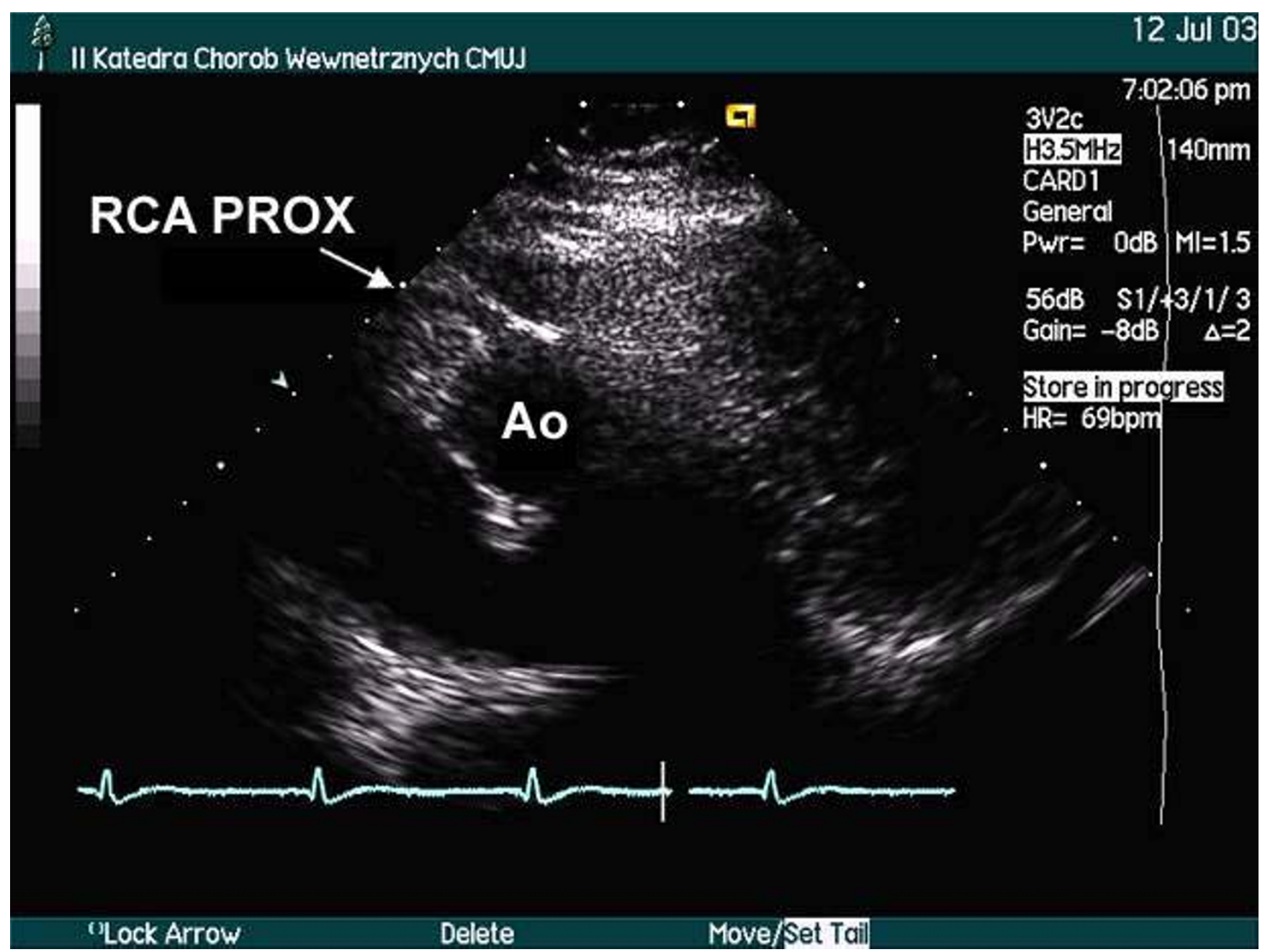

Figure 30

Proximal right coronary artery (RCA PROX) in parasternal short axis view. AO - aorta 


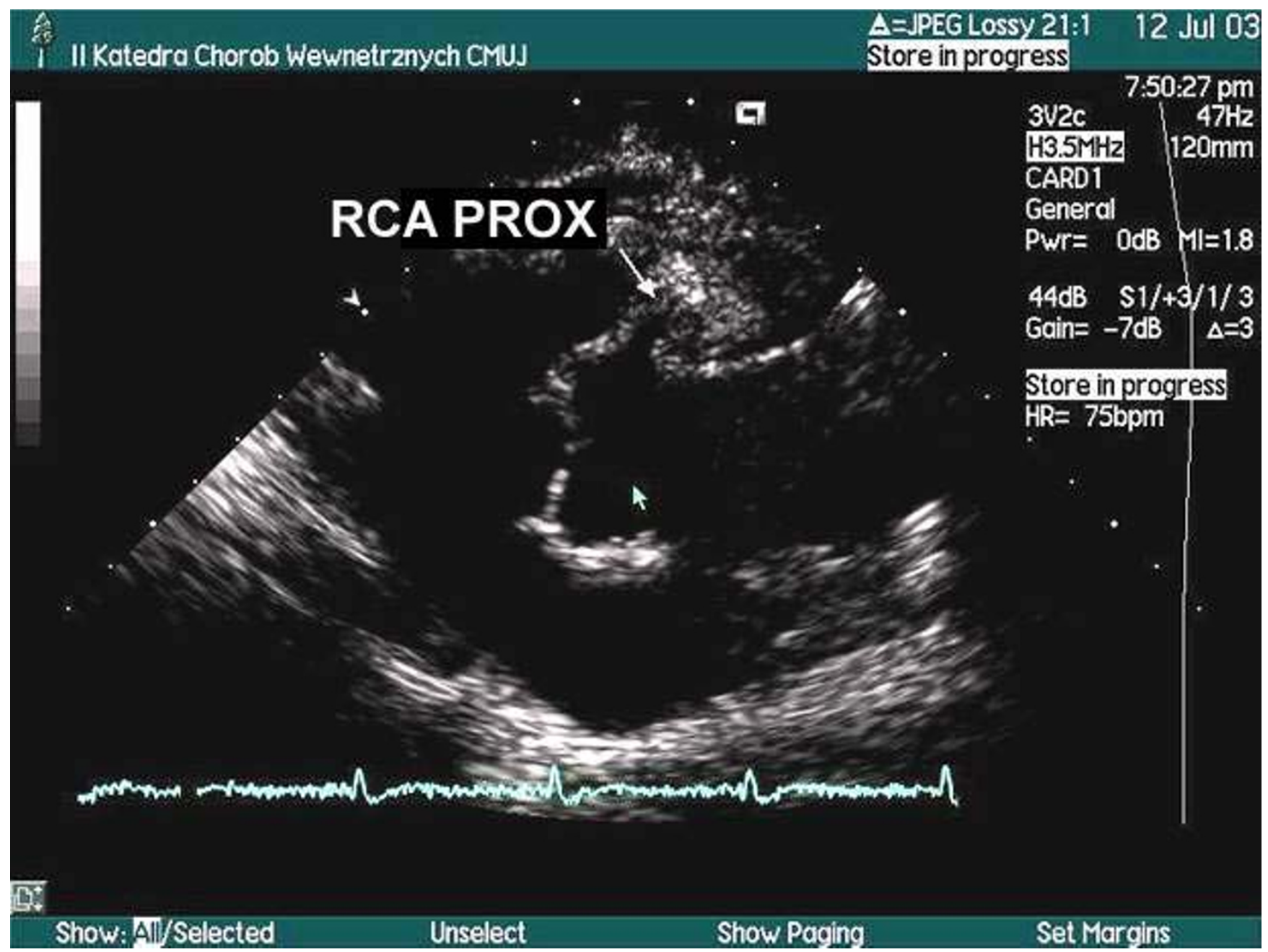

\section{Figure 3 I}

Proximal parts of the RCA (PROX RCA) in B - mode; modified parasternal long axis view 


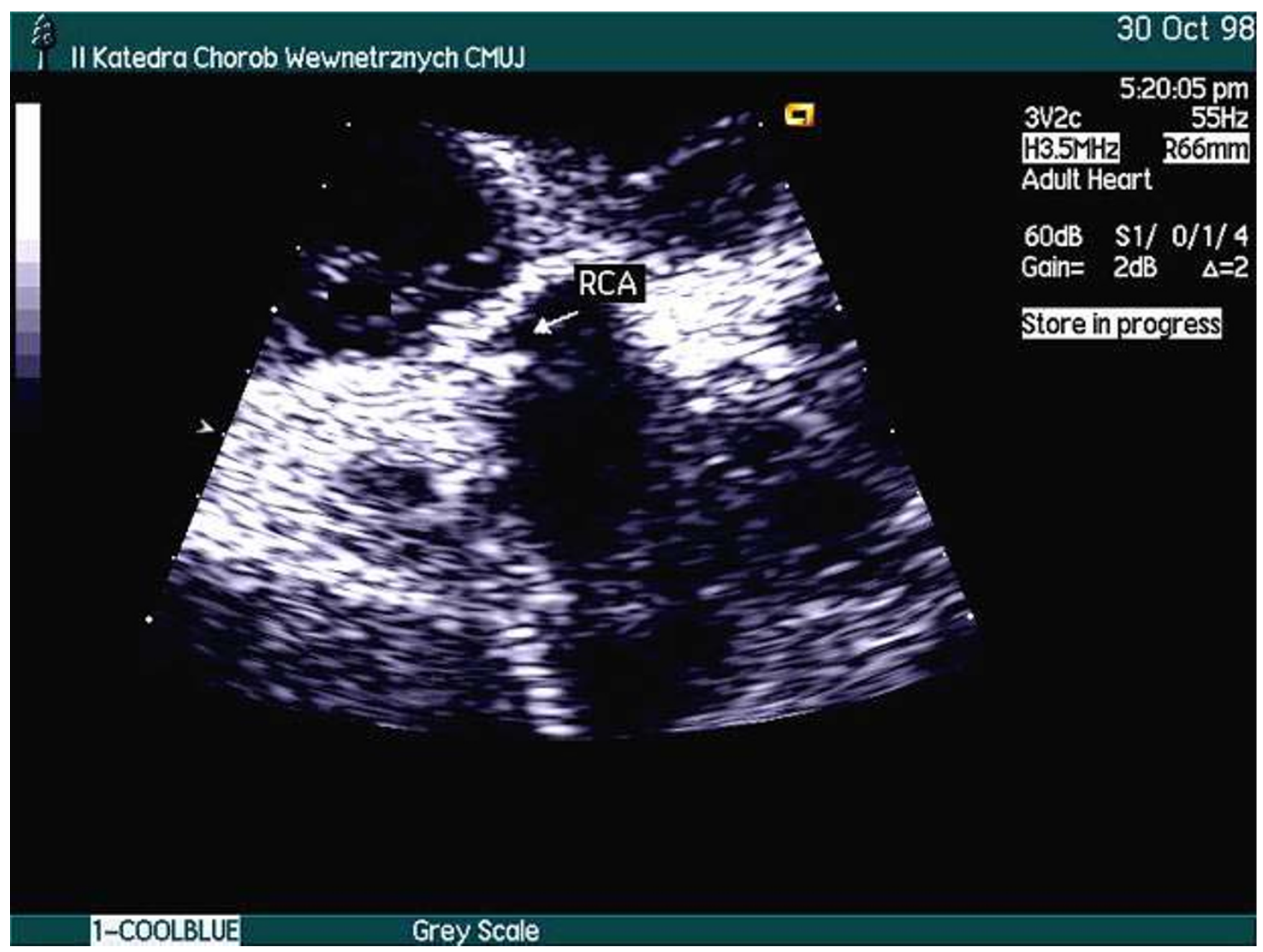

Figure 32

Proximal part of right coronary artery (RCA) in B-mode, modified apical 5-chamber view. 


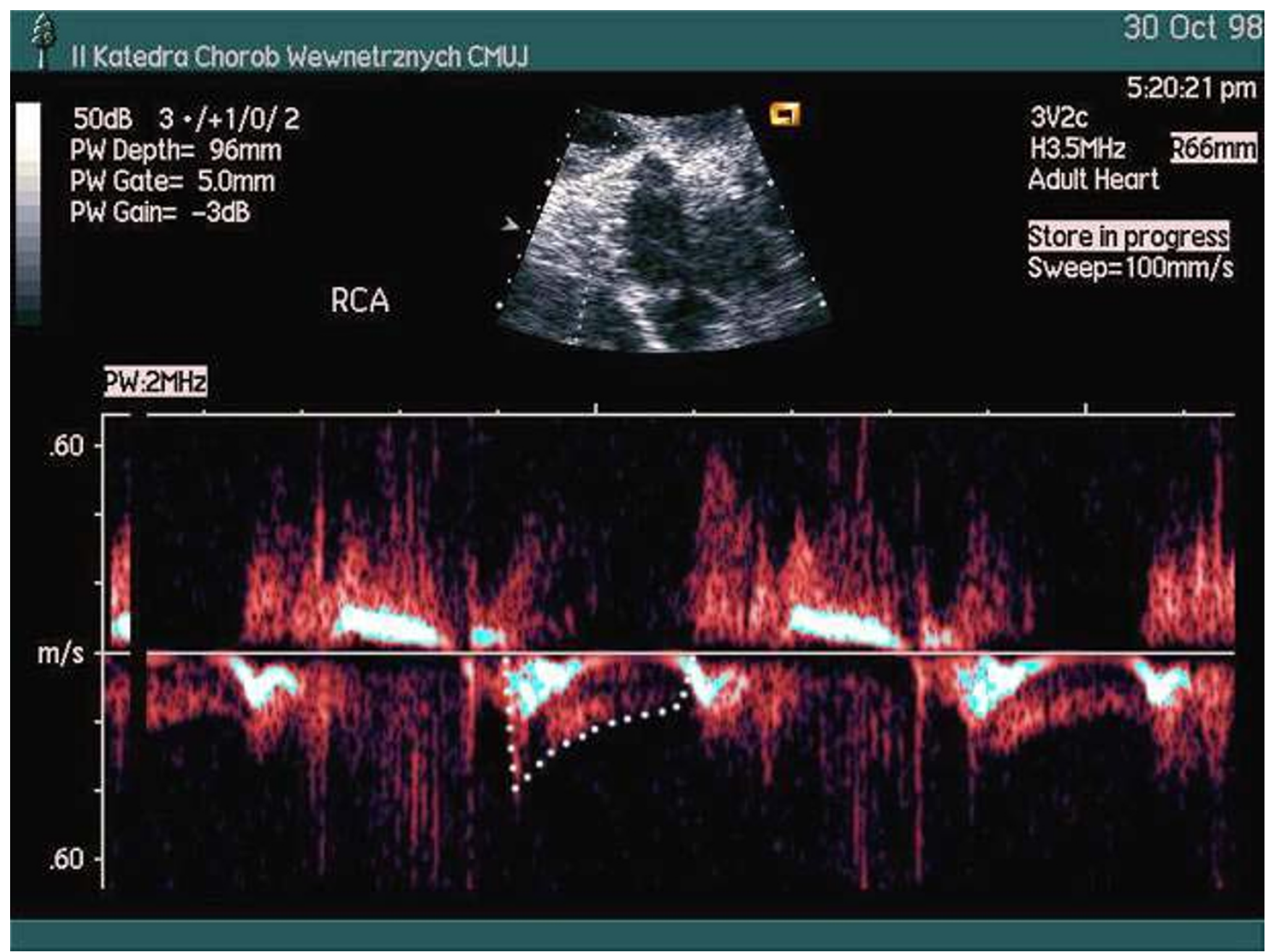

Figure 33

Duplex examination of proximal part of the right coronary artery (RCA) in modified apical 5-chamber view. 


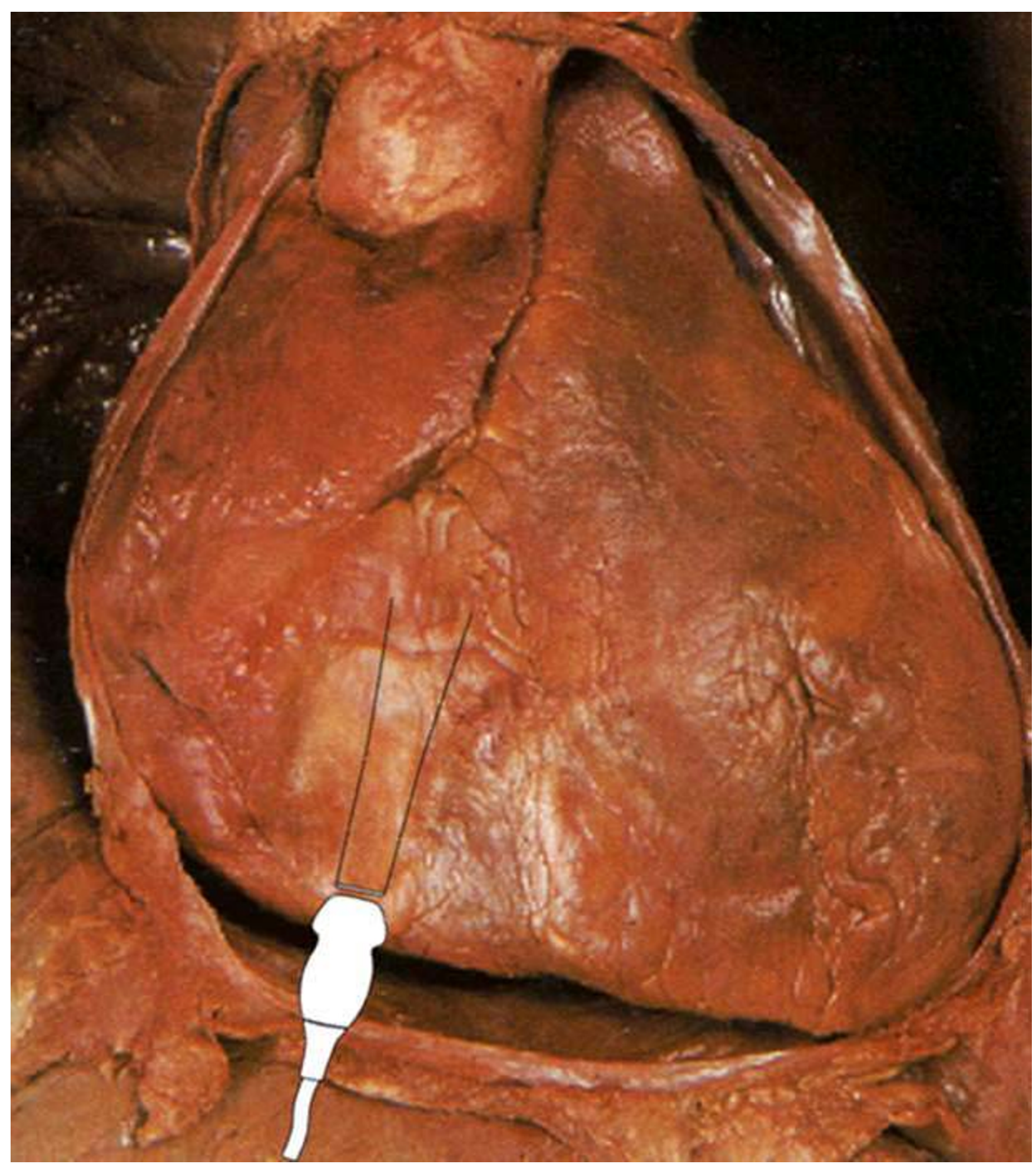

Figure 34

An artist drawing of the heart, view from the front. Spacial relations between ultrasound transducer in subcostal position and the heart are shown 


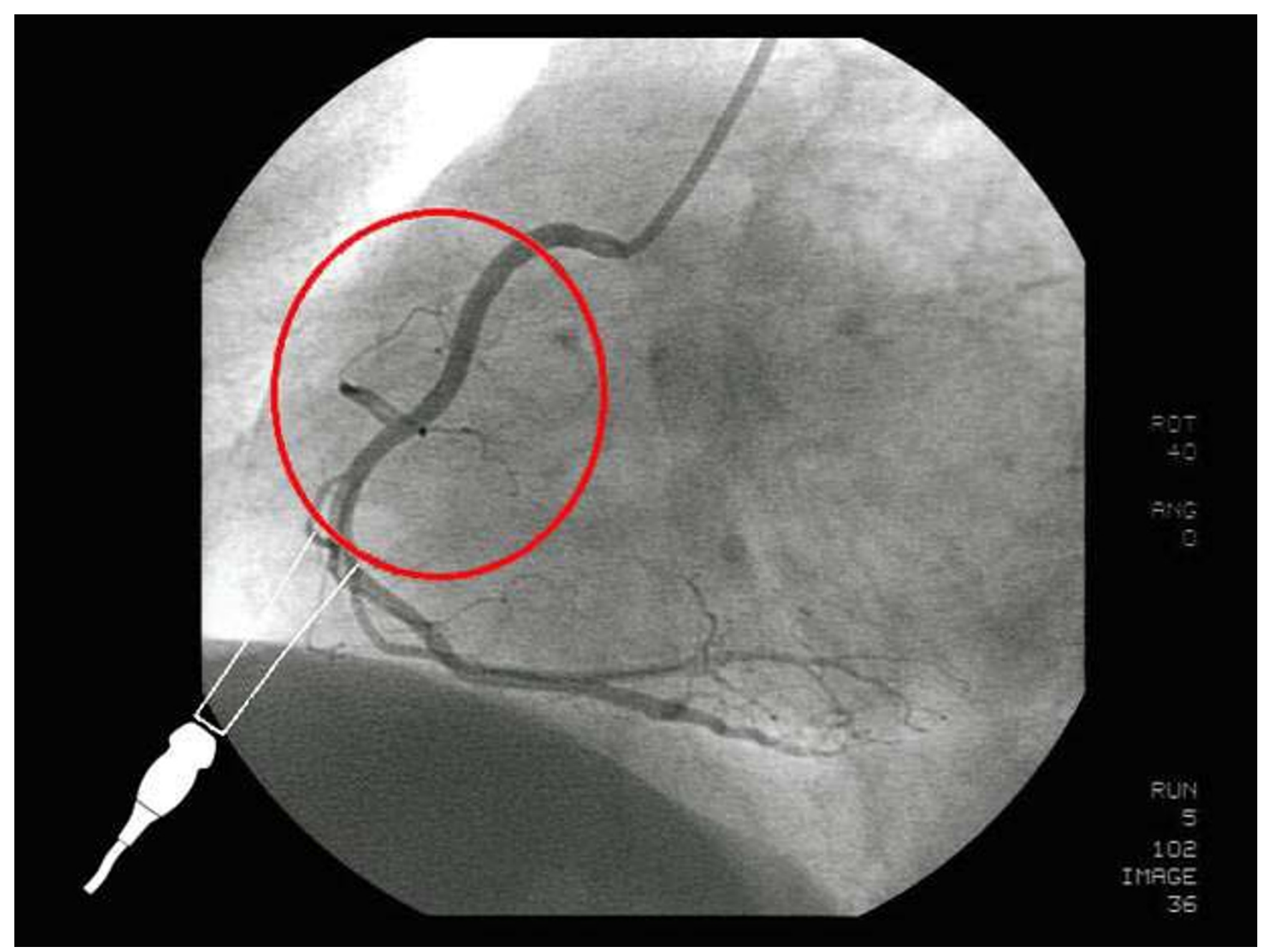

\section{Figure 35}

Angiography of the right coronary artery in LAO projection. Spacial relations between ultrasound transducer located in subcostal position and the middle segment of the right coronary artery (encircled in red) are shown 


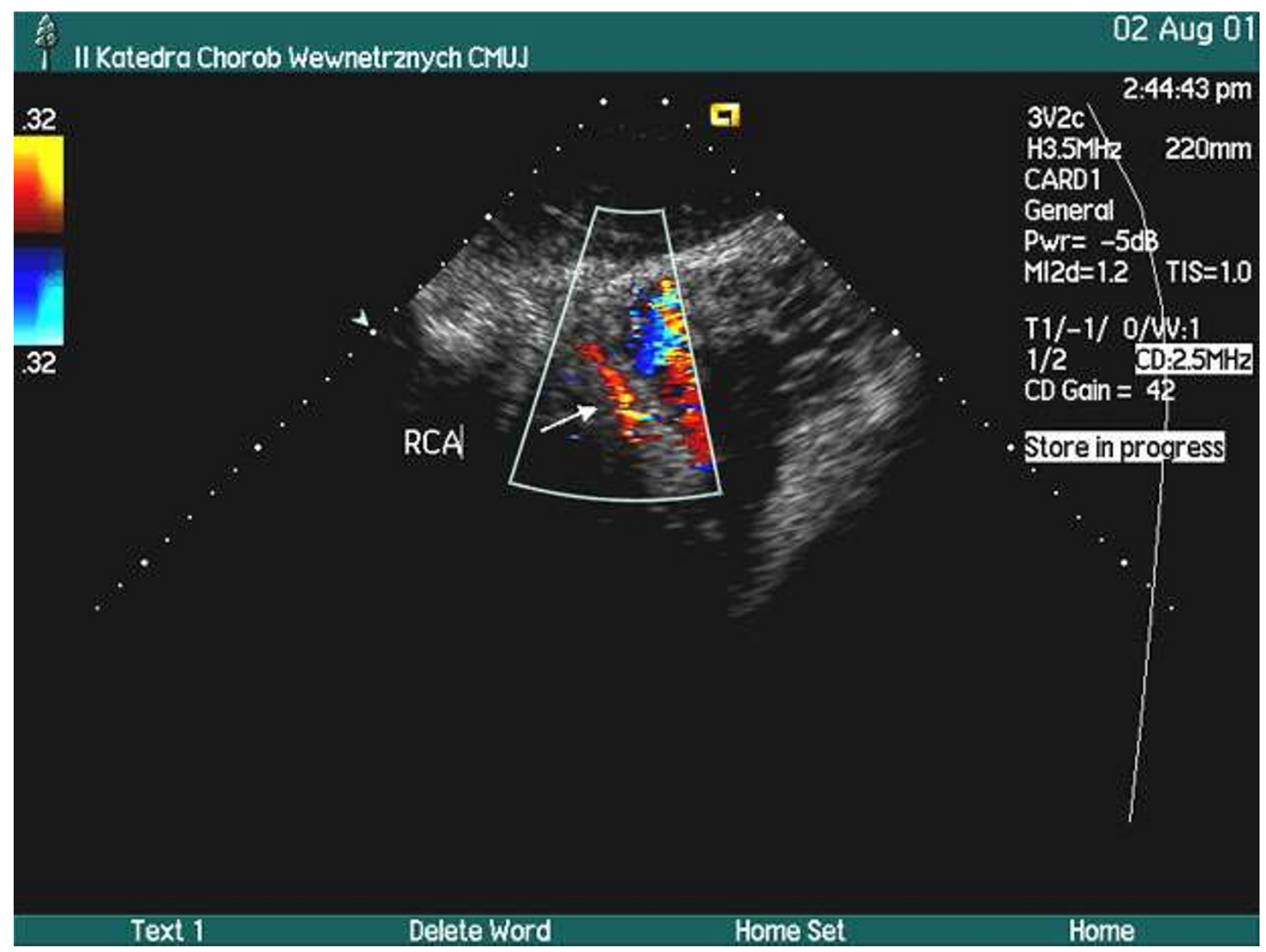

Figure 36

Middle segment of the RCA in color Doppler; modified subcostal short axis view See movie 7 [see Additional file 7] 


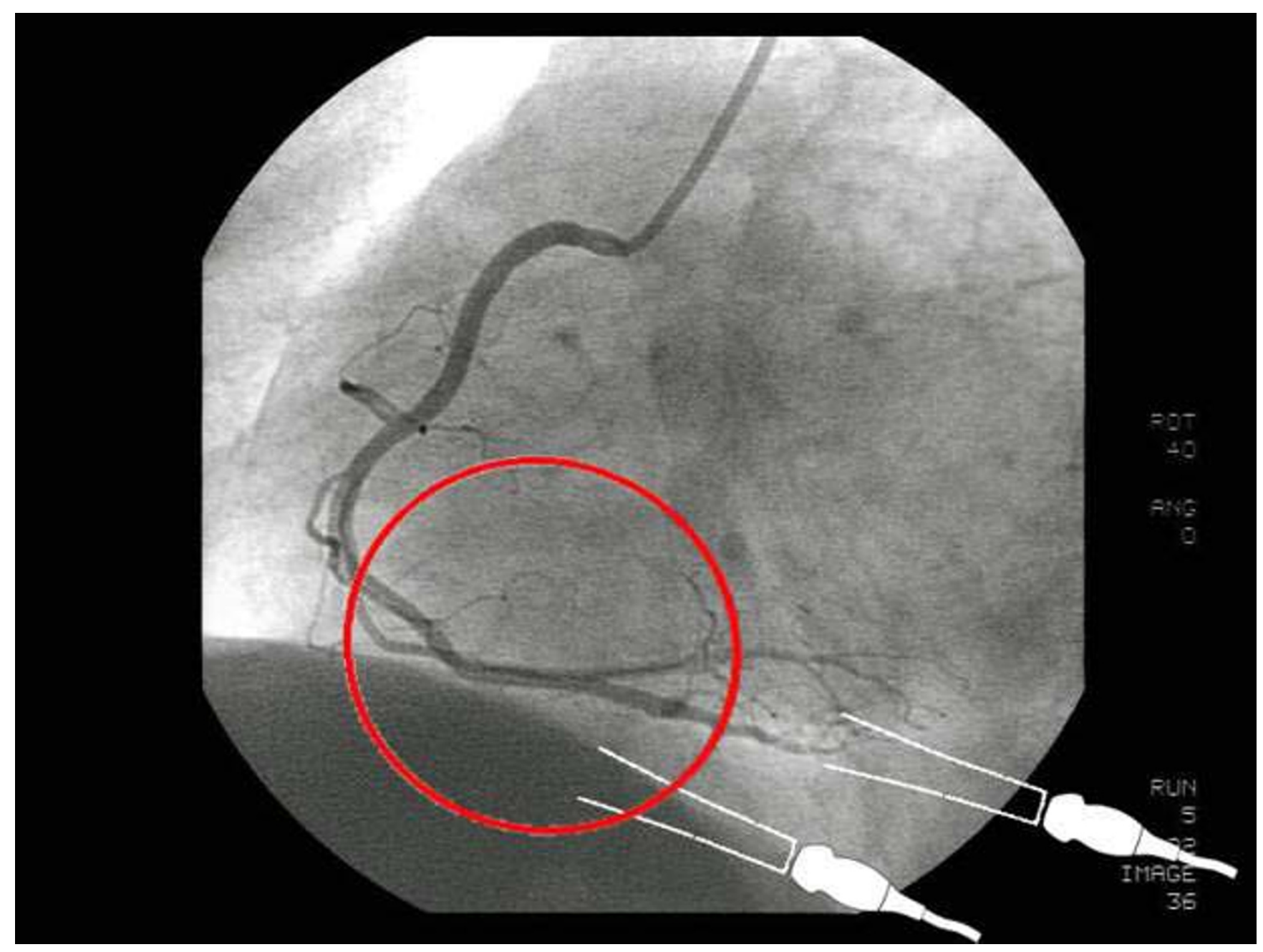

Figure 37

Angiography of the right coronary artery in LAO projection. Distal segment of the RCA at the inferior wall of the heart is marked by red circle for correlation with echocardiographic view 


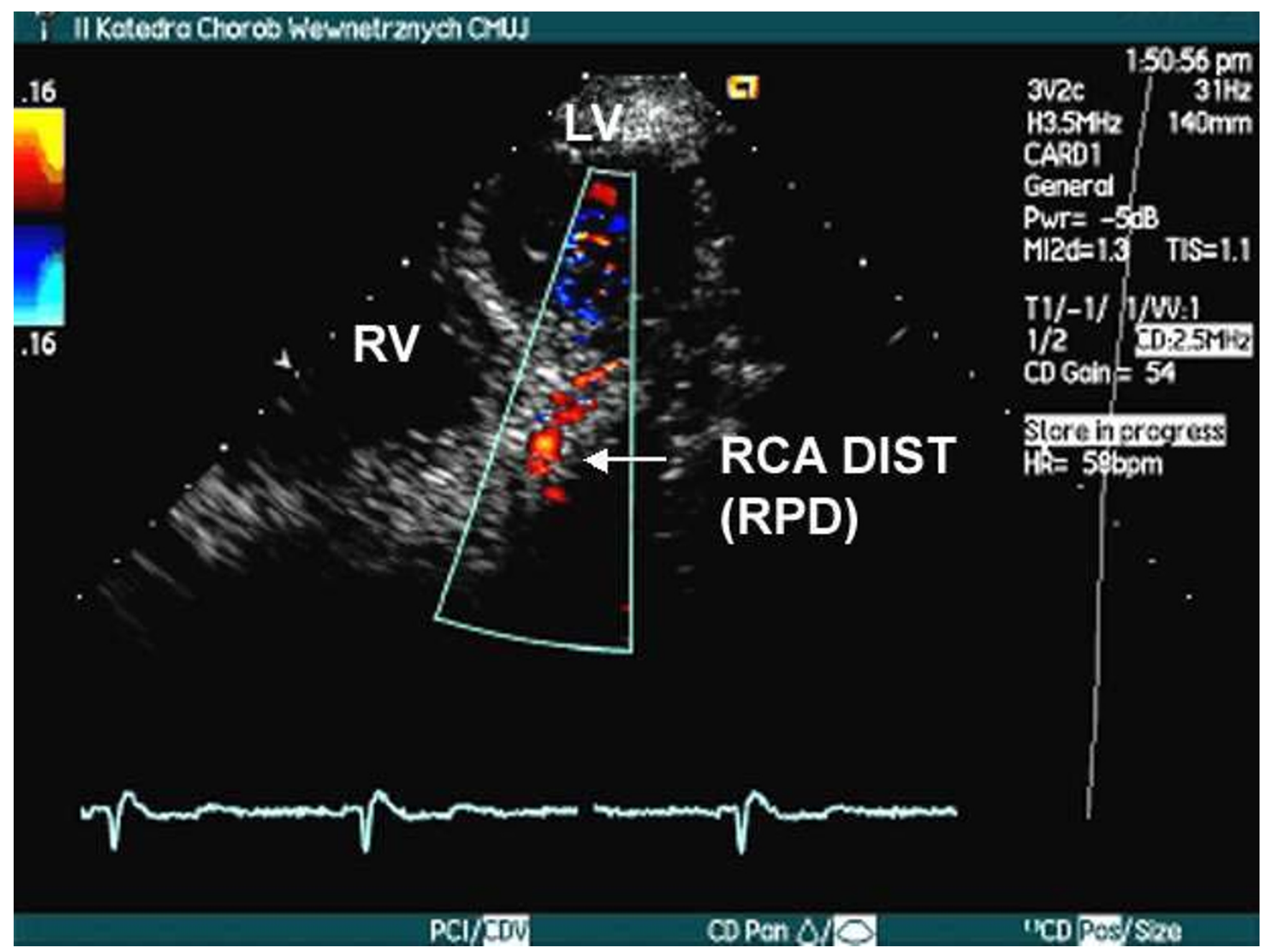

Figure 38

Distal RCA in color Doppler, modified apical 4-chamber view. See movie 8 [see Additional file Additional file 8] 


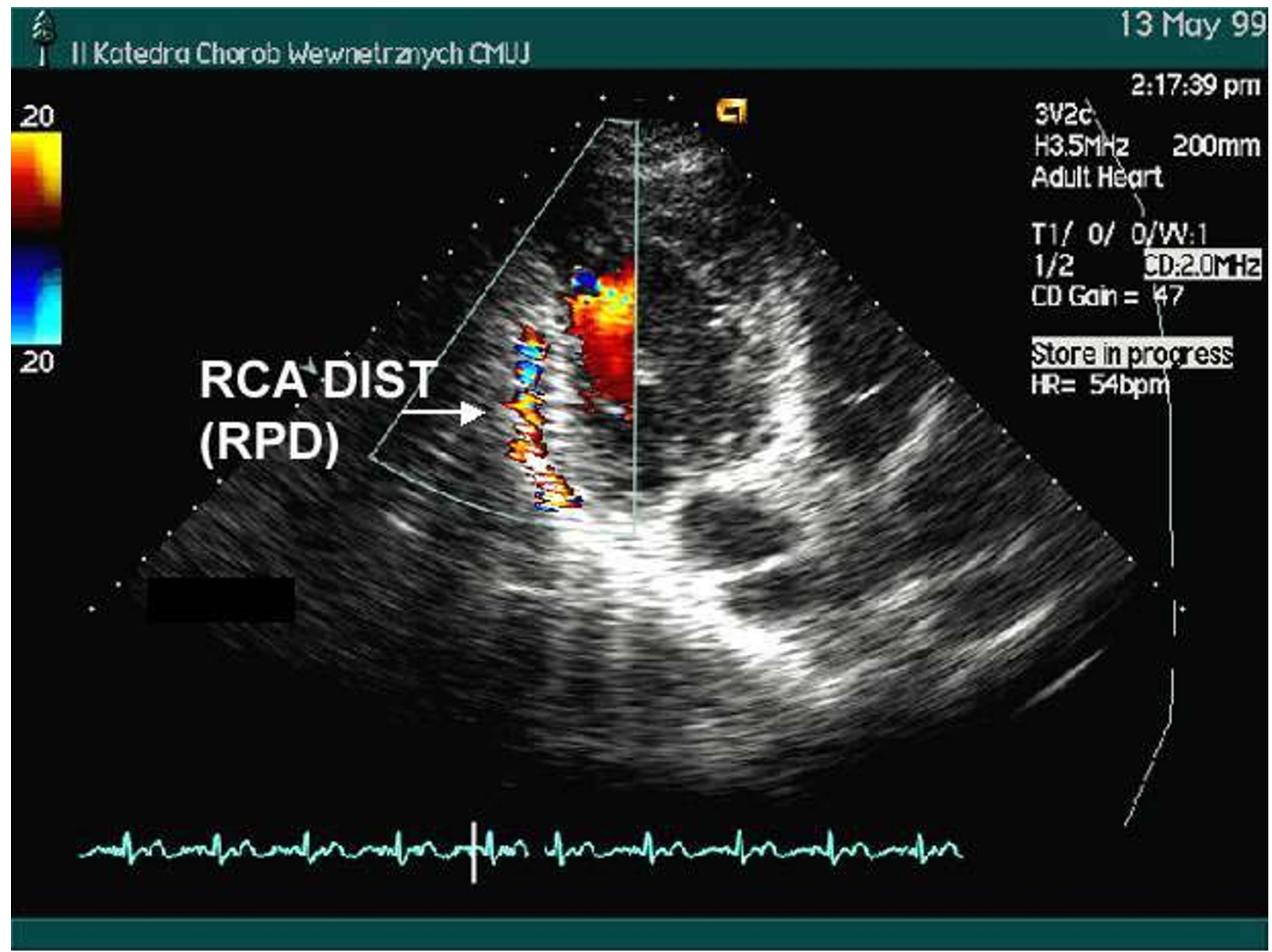

Figure 39

Distal RCA in color Doppler, modified apical 2-chamber view. 


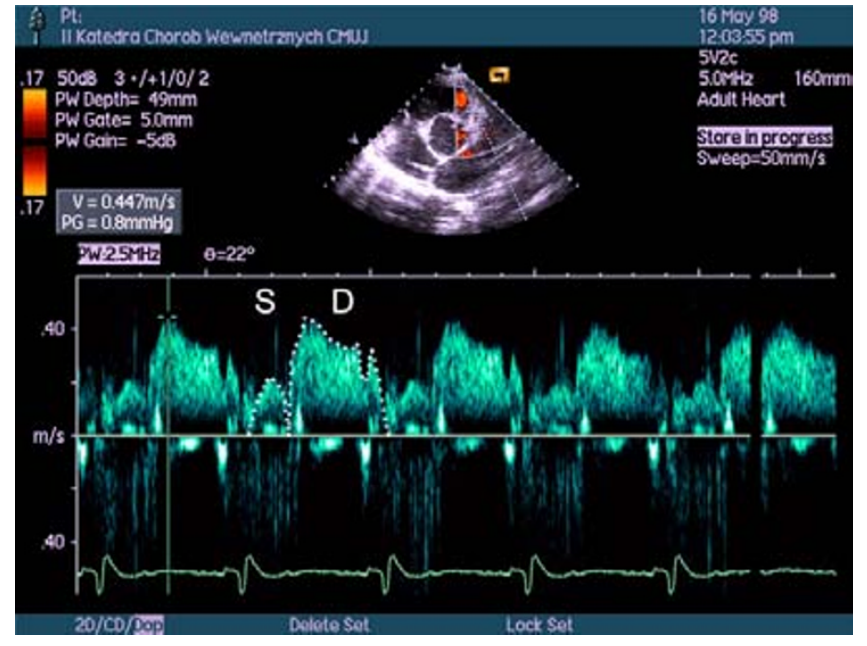

Figure 40

Duplex examination of middle segment of the LAD in modified parasternal long axis view. S - systolic, dome-like part of the flow velocity spectrum, D - diastolic part of the flow velocity spectrum, with typical, flame-like initial part

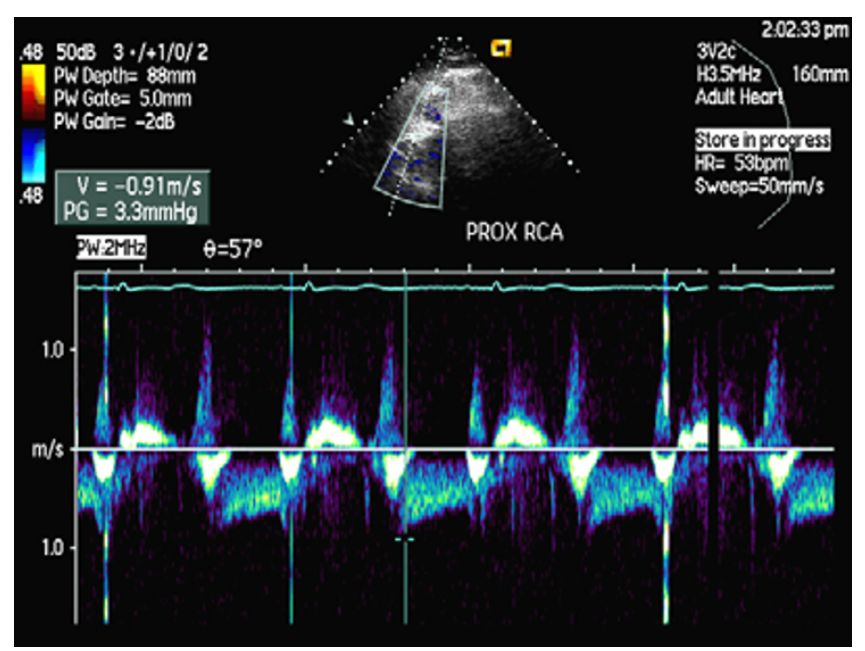

\section{Figure 4 I}

Duplex examination of proximal segment of the RCA in modified parasternal short axis view. Systolic part of the spectrum is not visible

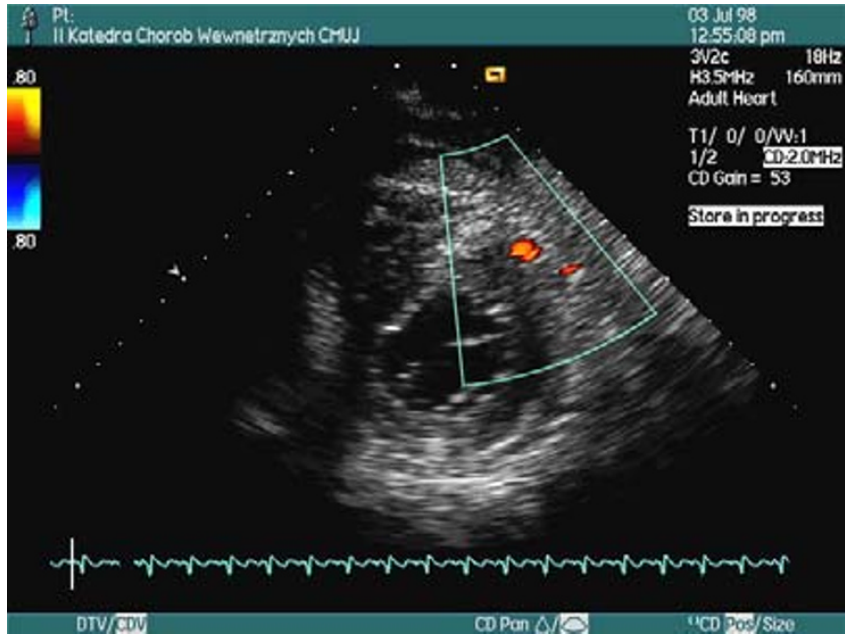

Figure 42

A bedside echocardiographic examination performed in a female patient 2 days after an acute anterior wall myocardial infarction. Parasternal short axis view at the level of papillary muscles. 2 spots of strong color signal were noted within the anterior wall; ... See movie 9 [see Additional file 9]

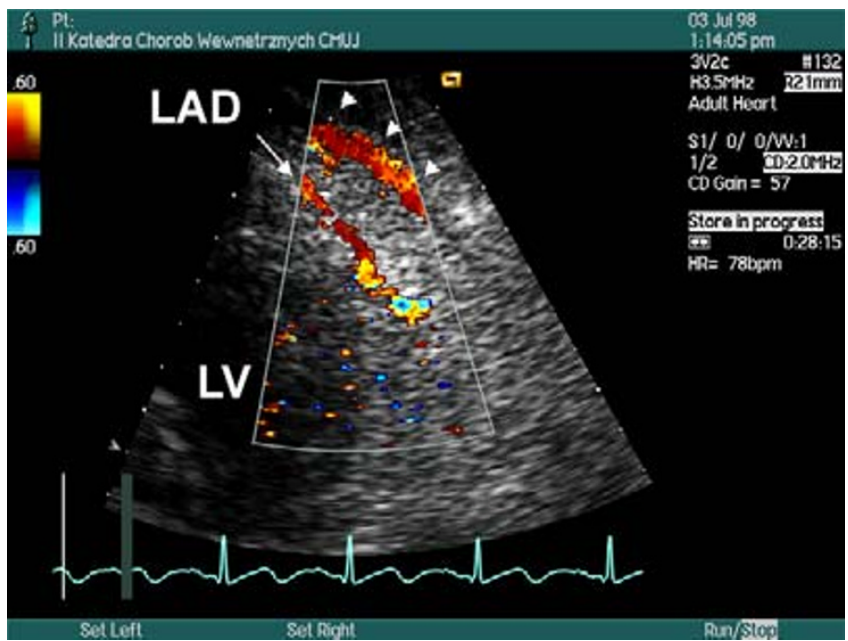

Figure 43

... after probe realignment a long segment of the LAD (arrow) with color aliasing corresponding to a local stenosis was visualized. Maximal flow velocity at the stenosis was 3.23 $\mathrm{m} / \mathrm{s}$. Please, note a strong signal of pericardial fluid flow (arrowheads). On subsequent coronary angiography critical stenoses of middle LAD and a diagonal branch were found 


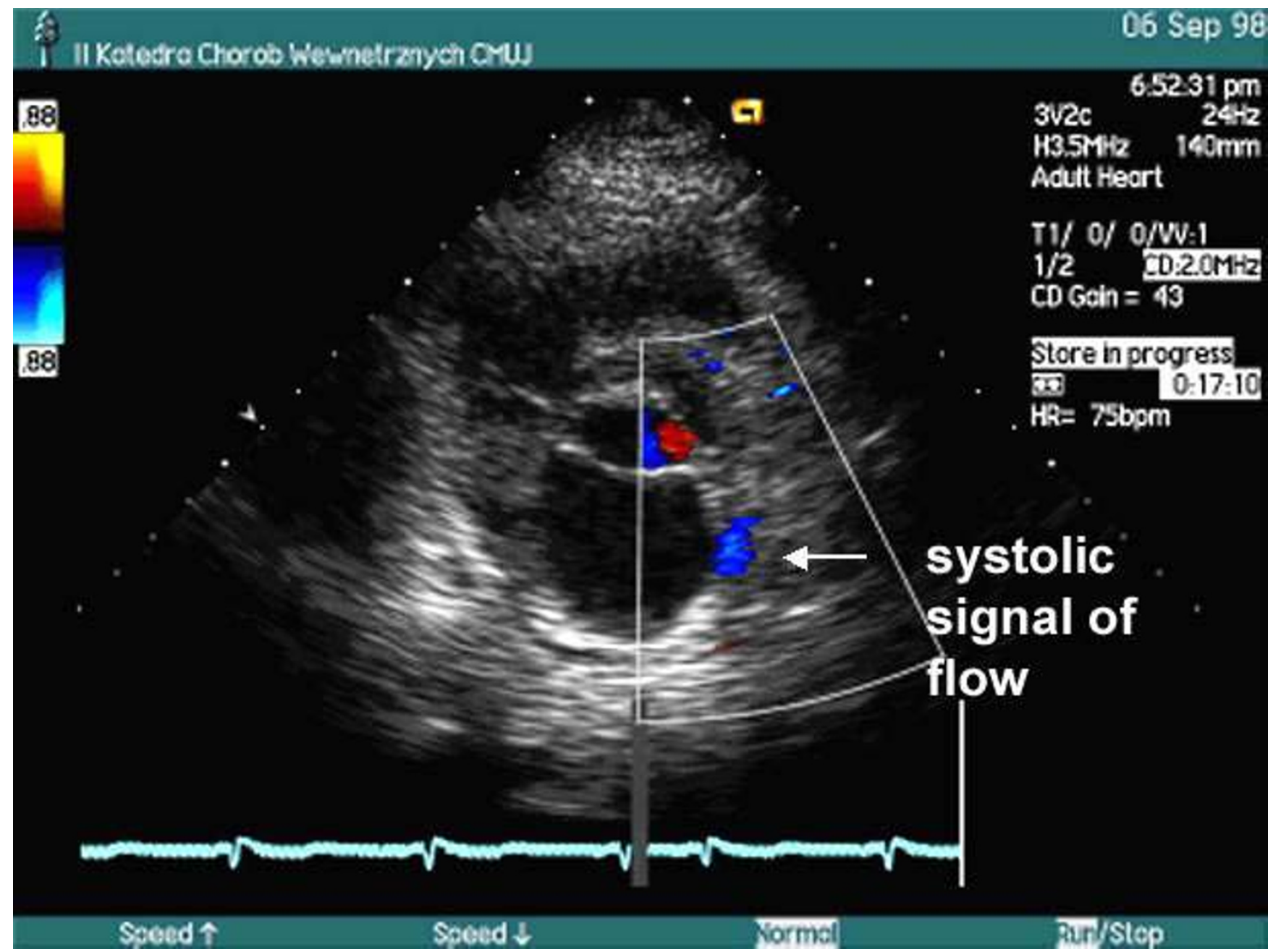

\section{Figure 44}

Color Doppler examination of left part of coronary sulcus in parasternal short axis view. Please, note, two separate color flow signals are seen: a systolic, seen more distally... 




\section{Figure 45}

... and a diastolic, seen more proximally. The systolic signal was most probably caused by flow of physiological amount of pericardial fluid, while the diastolic flow was related to the flow in the proximal Cx. See movie 10 [see Additional file 10] 


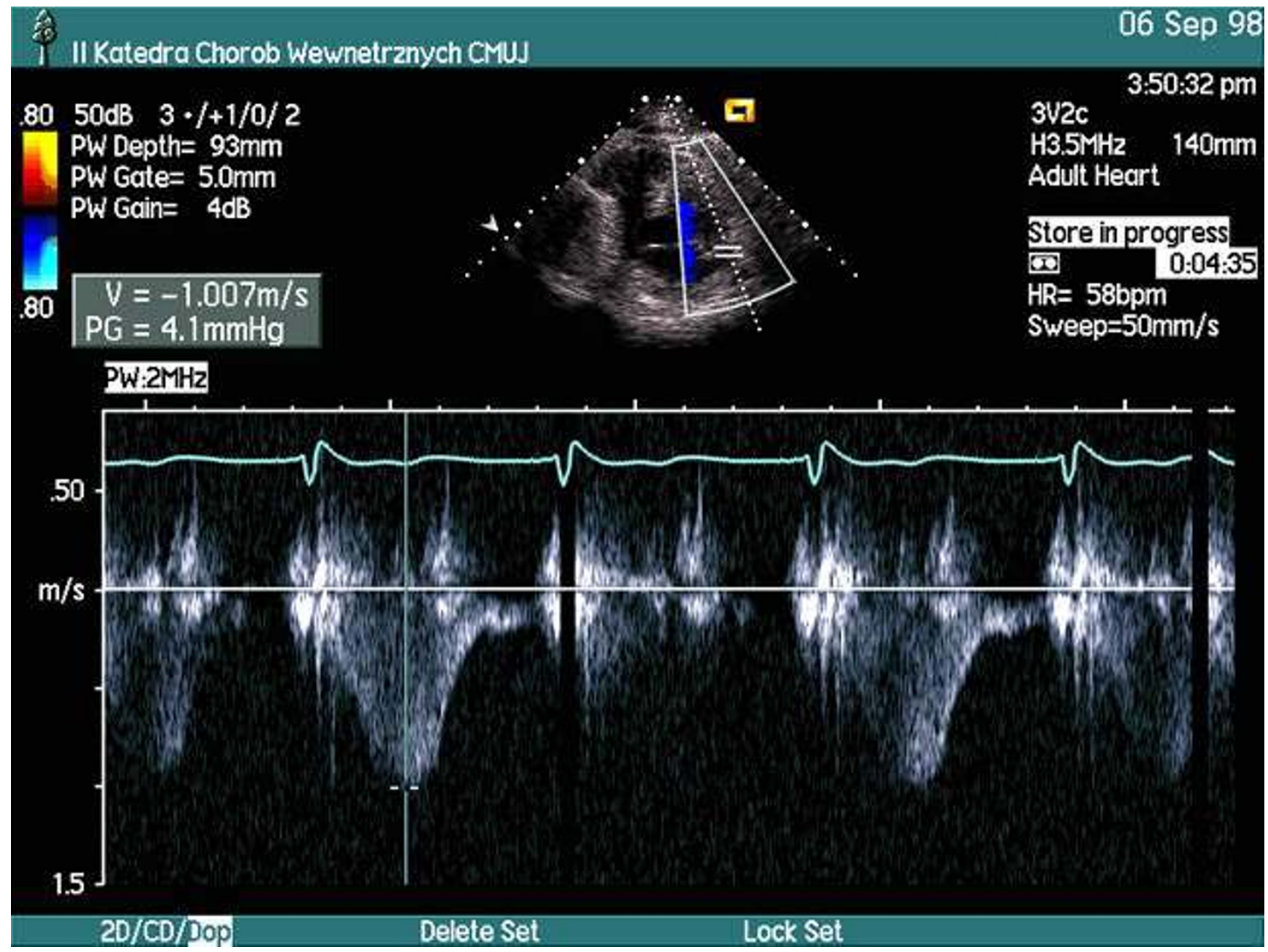

\section{Figure 46}

Duplex scan examination at the level of the more distal signal of flow. Strong, mostly systolic velocity spectrum is recorded, which further supports the diagnosis of flow of pericardial fluid 




\section{Figure 47}

Duplex scan examination performed at the more proximal signal of flow. Typical, predominantly diastolic flow within the Cx. 


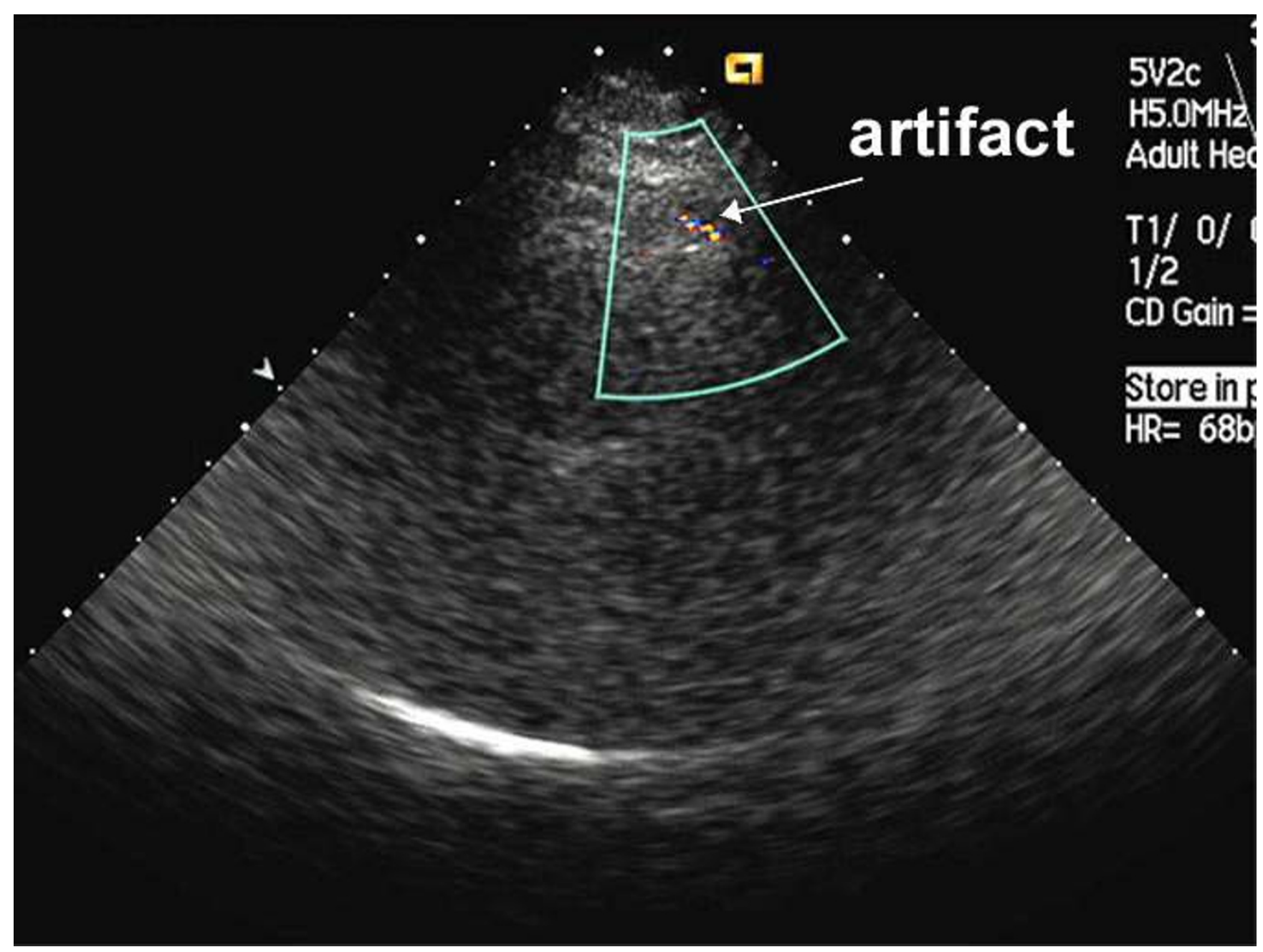

\section{Figure 48}

Doppler artifact at the anterior wall of the left ventricle. Color Doppler examination in the region of anterior interventricular groove, modified parasternal long axis view. Local, strong, linear signal mimicking high velocity flow is seen, which may easily be confused with tight middle LAD stenosis See movie II [see Additional file I I] 


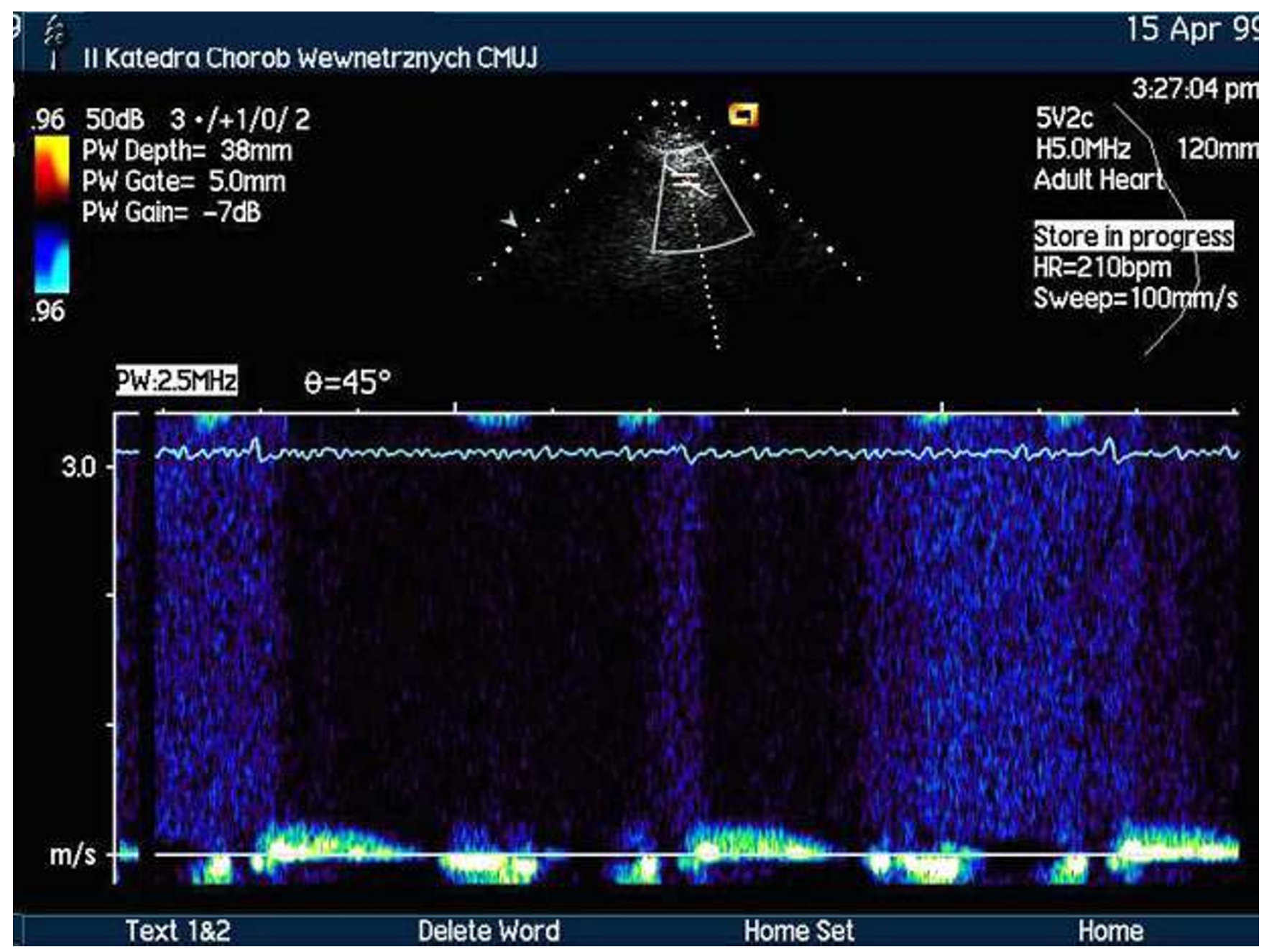

\section{Figure 49}

Doppler artifact at the anterior wall of the left ventricle, duplex examination within the area shown in Fig. 48. Strong signal, which is not confined to diastole is recorded. 
Table 2: Feasibility of harmonic mode transthoracic echocardiographic visualization of coronary arteries with regard to arterial segment (results are given as a ratio in which the numerator is a number arterial segments visualized and denominator - number of arterial segments studied) [9-II]

\begin{tabular}{lccc}
\hline \multicolumn{1}{c}{ Coronary artery } & Proximal segment & Middle segment & Distal segment \\
\hline LAD & & $35 / 50(70 \%) \#$ & $34 / 50(68 \%) \#$ \\
Ref. [10] & $34 / 50(68 \%) \#$ & $1 / 1 *$ \\
Ref. [II] & $20 / 20(100 \%)^{*}$ & $16 / 16(100 \%) *$ & - \\
Cx & $17 / 45(38 \%) \#$ & $11 / 45(24 \%) \#$ & - \\
Ref. [10] & $4 / 5 *$ & $2 / 3 *$ & - \\
Ref. [II] & $14 / 45(31 \%) \#$ & - & $15 / 45(33 \%) \#$ \\
RCA & $3 / 4 *$ & $5 / 15 *$ & $1 / 2 *$ \\
Ref. [10] & - & - & $62 / 81(76 \%) \#$ \\
Ref. [II] & & \\
Ref. [9] & & & \\
\hline
\end{tabular}

\# Not contrast enhanced TTE; patients scheduled for primary coronary angiography $[9,10] *$ Contrast enhanced TTE; patients scheduled for control coronary angiography [II] Abbreviations used in the table: LAD - left anterior descending coronary artery Cx - left circumflex coronary artery RCA - right coronary artery

Table 3: Accuracy of transthoracic echocardiographic diagnosis of coronary artery stenosis $(*)$ or restenosis (\#) in prospective studies of patients scheduled for primary (*) or control (\#) coronary angiography

\begin{tabular}{|c|c|c|c|c|}
\hline & Specificity & Sensitivity & PPV & NPV \\
\hline Ref. $[10] *$ & $96-100 \%$ & $62-66 \%$ & $82-100 \%$ & $86-91 \%$ \\
\hline Ref. [22] (\#) & $93 \%$ & $86 \%$ & & \\
\hline Ref. [II] (\#) & $94 \%$ & $100 \%$ & $80 \%$ & $100 \%$ \\
\hline
\end{tabular}




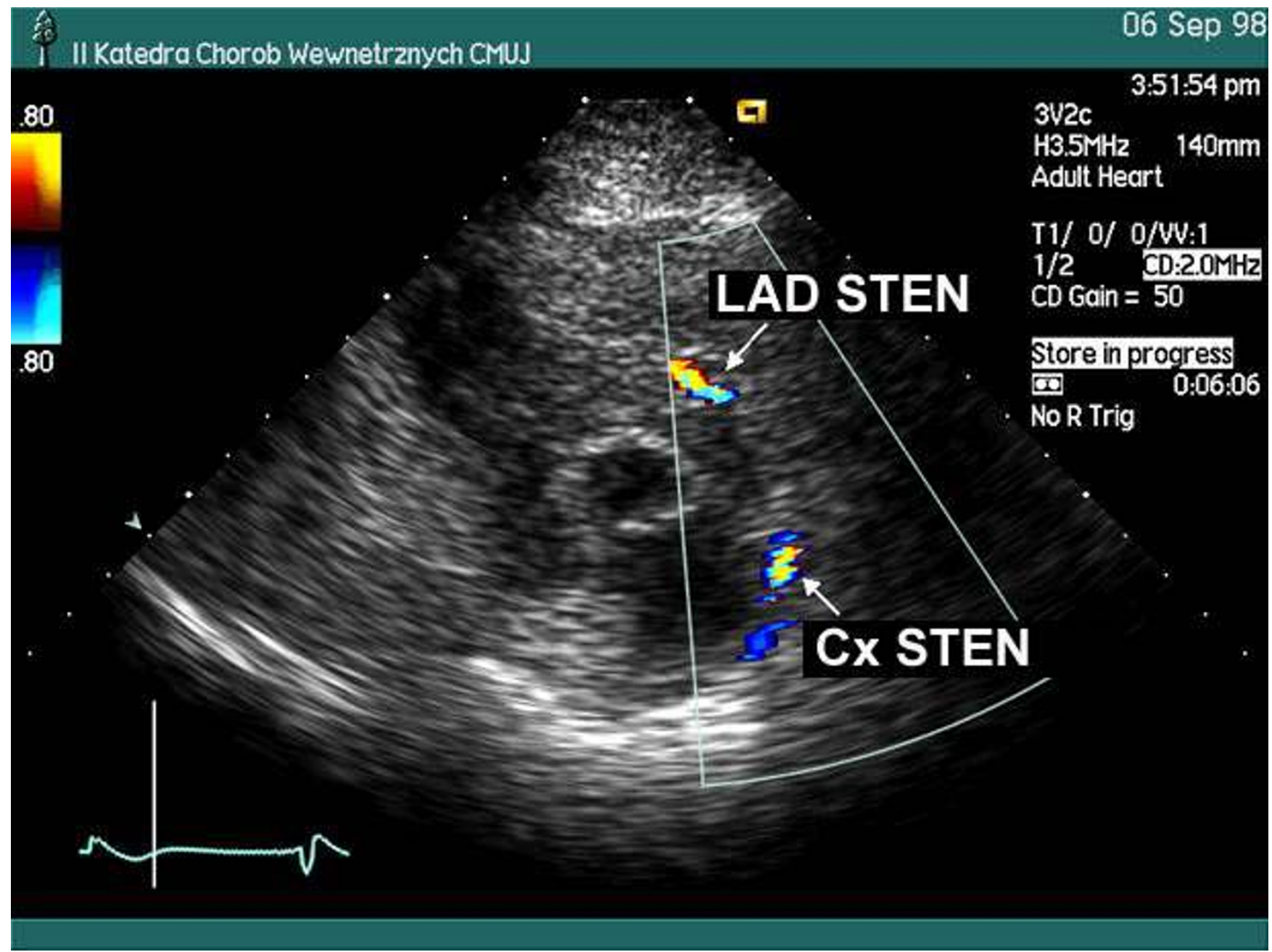

\section{Figure $\mathbf{5 0}$}

Color Doppler transthoracic echocardiography, parasternal short axis view. Proximal LAD and proximal Cx stenoses were incidentally found on rutine echocardiographic examination. They were later confirmed by coronary angiography. See movie $I 2$ [see Additional file 12] 


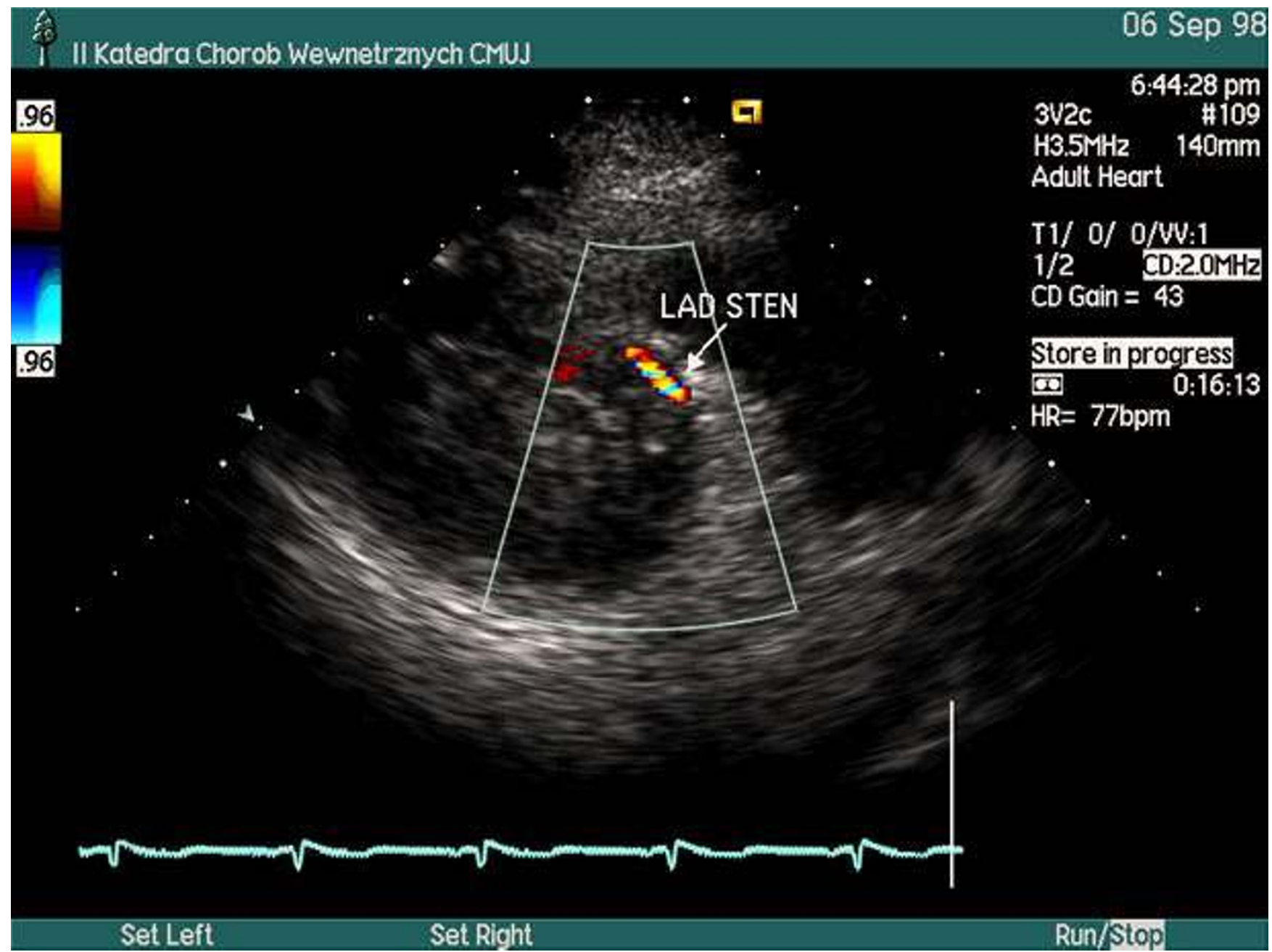

\section{Figure 5 I}

Color Doppler echocardiography, parasternal modified short axis view. Middle LAD stenosis was incidentally found on rutine TTE. It was later confirmed by coronary angiography. See movie I 3 [see Additional file I3] 


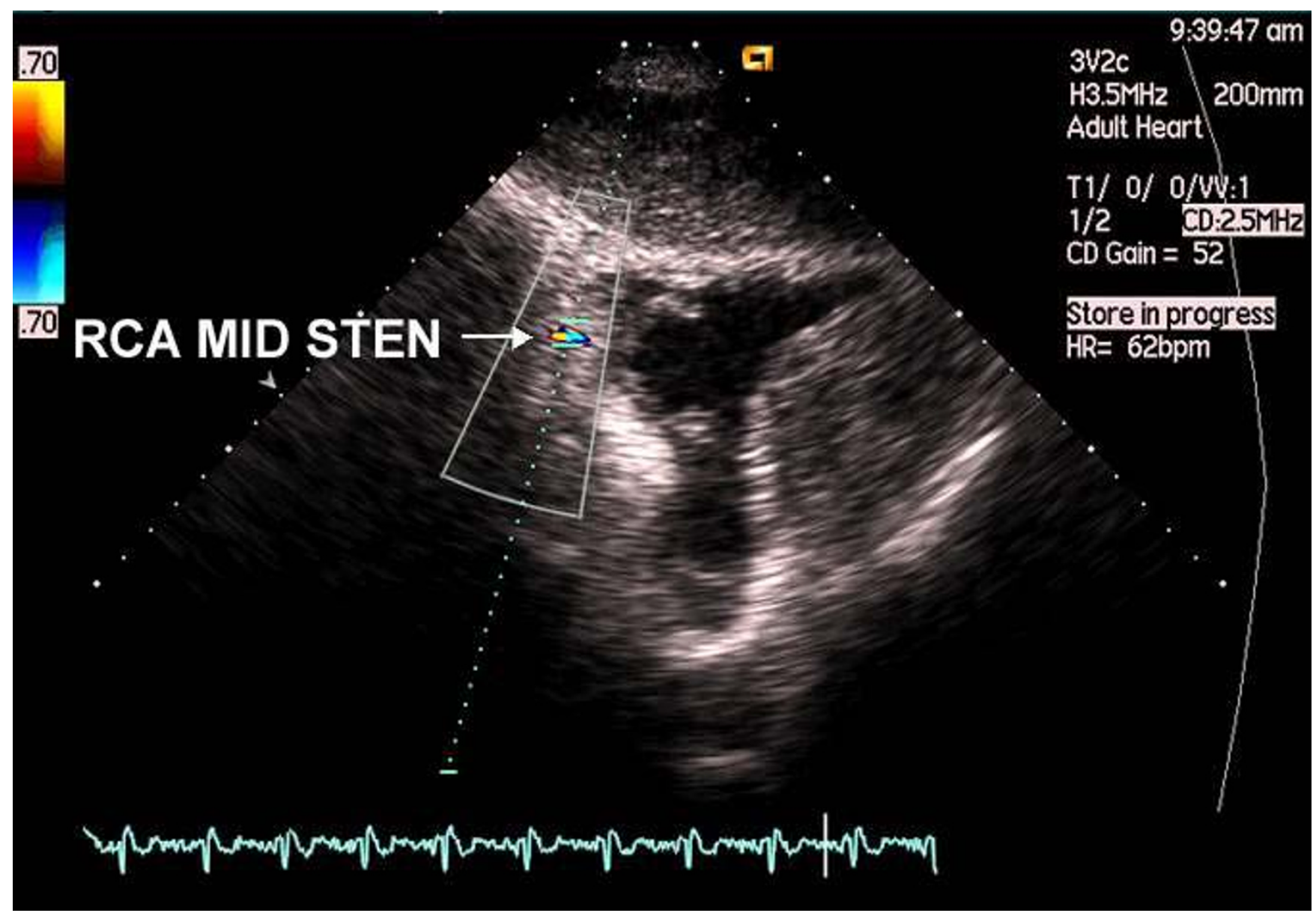

\section{Figure $\mathbf{5 2}$}

Color Doppler examination of middle RCA, modified subcostal short axis view. Please, note local color aliasing in distal part of coronary sulcus is seen. 




Figure 53

Color Doppler examination of middle RCA, modified subcostal short axis view. Normal color Doppler signal seen proximally to the area depicted in Fig. 52. 


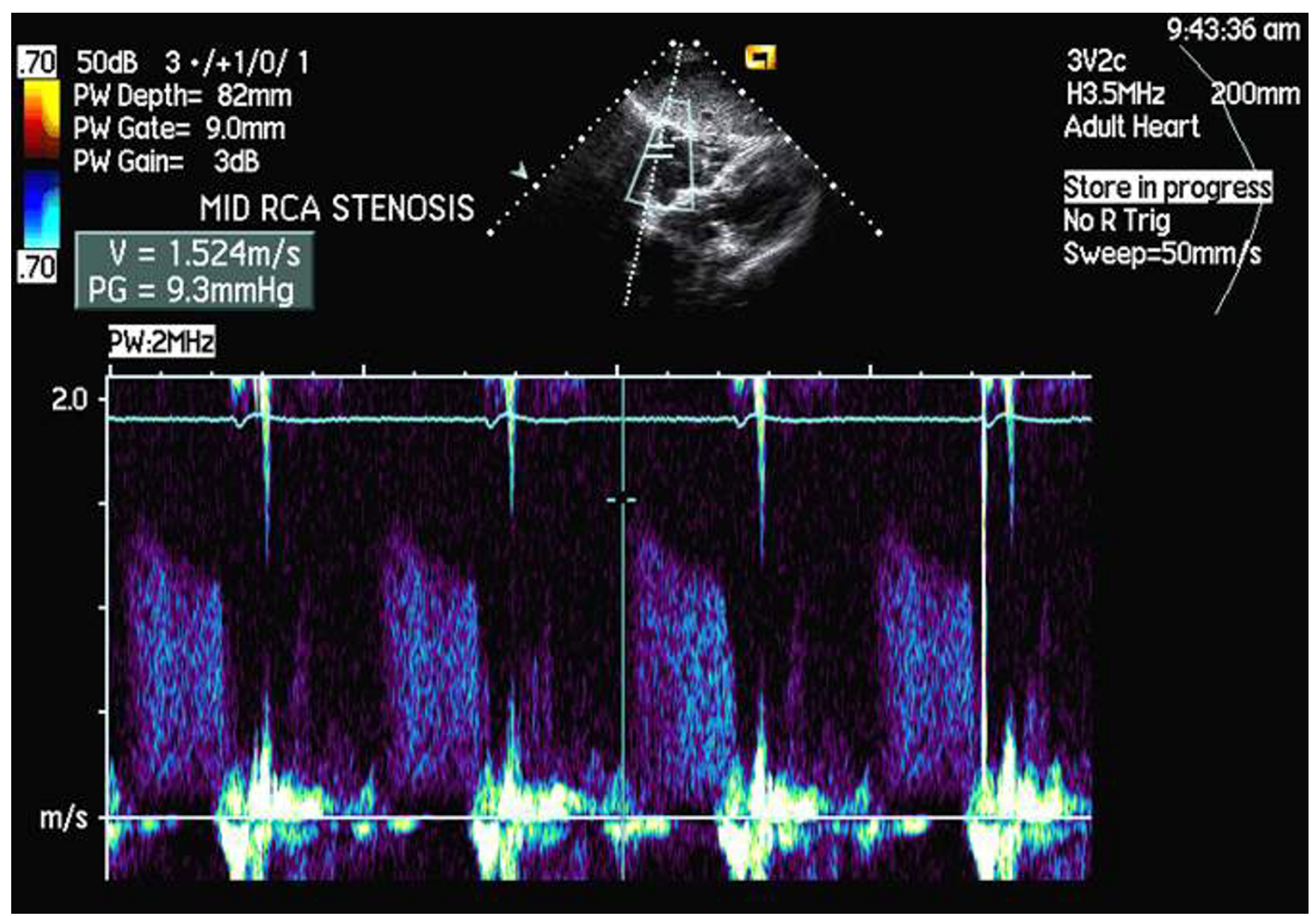

Figure 54

Duplex ultrasound performed at the level of color aliasing. Maximal flow velocity of $1.52 \mathrm{~m} / \mathrm{s}$ was recorded. 


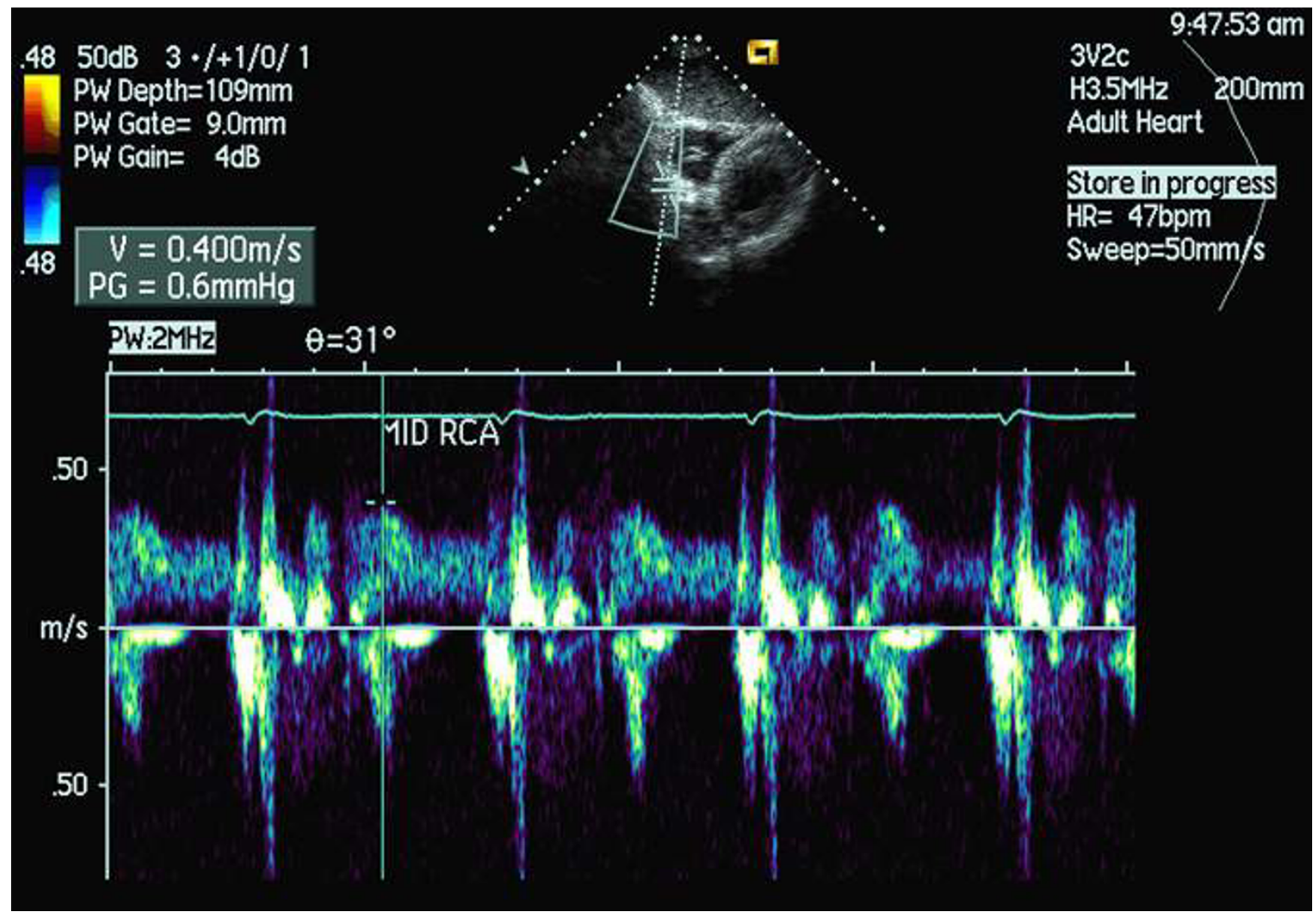

Figure 55

Duplex ultrasound performed within area shown in Fig. 53. Maximal flow velocity of $0,4 \mathrm{~m} / \mathrm{s}$ was recorded. Maximal flow velocity ratio (velocity within stenosis divided by velocity proximal to stenosis) was 3.8 proving hemodynamic significance of the stenosis. Tight middle RCA stenosis was later found on coronary angiography.

\section{Additional material}

\section{Additional File 1}

Movie 1. Proximal part of left coronary artery in B-mode, parasternal short axis view. LAD PROX - proximal part of the left anterior descending coronary artery, INTERMEDIATE - strong intermediate branch, $C x$ $P R O X$ - proximal part of left circumflex coronary artery (for best results play the movie in an endless loop)

Click here for file

[http://www.biomedcentral.com/content/supplementary/14767120-1-16-S1.mov]

\section{Additional File 2}

Movie 2. Proximal part of left coronary artery in color Doppler, parasternal short axis view. Origin of left circumflex coronary artery is also seen (for best results play the movie in an endless loop)

Click here for file

[http://www.biomedcentral.com/content/supplementary/14767120-1-16-S2.mov]

\section{Additional File 3}

Movie 3. Middle LAD in color Doppler. Modified long axis view (for best results play the movie in an endless loop)

Click here for file

[http://www.biomedcentral.com/content/supplementary/1476-

7120-1-16-S3.mov]

\section{Additional File 4}

Movie 4. Proximal left circumflex, left main, proximal left anterior descending coronary arteries in color Doppler, prarasternal short axis view. Courtesy of Acuson Corp. (for best results play the movie in an endless loop)

Click here for file

[http://www.biomedcentral.com/content/supplementary/14767120-1-16-S4.mov] 


\section{Additional File 5}

Movie 5. Middle part of left circumflex coronary artery in color Doppler. Parasternal short axis view, imaging plane traversing coronary sulcus. The flow in artery is depicted in blue. (for best results play the movie in an endless loop)

Click here for file

[http://www.biomedcentral.com/content/supplementary/14767120-1-16-S5.mov]

\section{Additional File 6}

Movie 6. Middle part of left circumflex coronary artery in color Doppler. Modified parasternal long axis view, imaging plane traversing lateral wall of the left ventricle. The flow in artery is depicted in blue. (for best results play the movie in an endless loop)

Click here for file

[http://www.biomedcentral.com/content/supplementary/14767120-1-16-S6.mov]

\section{Additional File 7}

Movie 7. Middle segment of the RCA in color Doppler; modified subcostal short axis view (for best results play the movie in an endless loop) Click here for file [http://www.biomedcentral.com/content/supplementary/14767120-1-16-S7.mov]

\section{Additional File 8}

Movie 8. Distal RCA in color Doppler, modified apical 4-chamber view. (for best results play the movie in an endless loop)

Click here for file

[http://www.biomedcentral.com/content/supplementary/14767120-1-16-S8.mov]

\section{Additional File 9}

Movie 9. A female patient in 2 days after an acute anterior wall myocardial infarction. Parasternal short axis view at the level of papillary muscles. 2 spots of strong color signal were noted within the anterior wall, which correspond to tight stenoses of the midlle LAD and a diagonal branch (for best results play the movie in an endless loop) Click here for file [http://www.biomedcentral.com/content/supplementary/14767120-1-16-S9.mov]

\section{Additional File 10}

Movie 10. Color Doppler examination of left part of coronary sulcus in parasternal short axis view. Please, note, two separate color flow signals are seen: a systolic, seen more distally, and a diastolic, seen more proximally. The systolic signal is most probably caused by flow of physiological amount of pericardial fluid, while the diastolic flow is related to the flow in the proximal $C x$. (for best results play the movie in an endless loop) Click here for file

[http://www.biomedcentral.com/content/supplementary/14767120-1-16-S10.mov]

\section{Additional File 11}

Movie 11. Doppler artifact at the anterior wall of the left ventricle. Color Doppler examination in the region of anterior interventricular groove, modified parasternal long axis view. Local, strong, linear signal mimicking high velocity flow is seen, which may easily be confused with tight middle LAD stenosis (for best results play the movie in an endless loop) Click here for file

[http://www.biomedcentral.com/content/supplementary/14767120-1-16-S11.mov]

\section{Additional File 12}

Movie 12. Color Doppler transthoracic echocardiography, parasternal short axis view. Proximal $L A D$ and proximal $C x$ stenoses were incidentally found on rutine echocardiographic examination. They were later confirmed by coronary angiography. (for best results play the movie in an endless loop)

Click here for file

[http://www.biomedcentral.com/content/supplementary/14767120-1-16-S12.mov]

\section{Additional File 13}

Movie 13. Color Doppler echocardiography, parasternal modified short axis view. Middle LAD stenosis was incidentally found on rutine TTE. It was later confirmed by coronary angiography. (for best results play the movie in an endless loop)

Click here for file

[http://www.biomedcentral.com/content/supplementary/1476-

7120-1-16-S13.mov]

\section{References}

I. Caiati C, Montaldo C, Zedda N, Bina A, lliceto S: New noninvasive method for coronary flow reserve assessment. Contrastenhanced transthoracic second harmonic echo Doppler. Circulation 1999, 99:77।-778.

2. Kenny A, Shapiro LM: Transthoracic high-frequency twodimensional echocardiography, Doppler and color flow mapping to determine anatomy and blood flow patterns in the distal left anterior descending coronary artery. Am J Cardiol 1992, 69:1265-8.

3. Hozumi T, Yoshida K, Ogata Y, Akasaka T, Asami Y, Takagi T, Morioka S: Noninvasive assessment of significant left anterior descending coronary artery stenosis by coronary flow velocity reserve with transthoracic color Doppler echocardiography. Circulation 1998, 97:1557-62.

4. Lambertz $H$, Tries HP, Stein T, Lethen $H$ : Noninvasive assessment of coronary flow reserve with transthoracic signal-enhanced Doppler echocardiography. J Am Soc Echocardiogr 1999, 12:186-195.

5. Eleuteri E, Scapellato F, Temporelli PL, Giannuzzi P: Evaluation of the left anterior descending coronary artery flow velocity by transthoracic echo-Doppler without contrast enhancement. Ital Heart J 2002, 3:520-4.

6. Okayama H, Sumimoto T, Hiasa G, Morioka N, Yamamoto K, Kawada $\mathrm{H}$ : Usefulness of an echo-contrast agent for assessment of coronary flow velocity and coronary flow velocity reserve in the left anterior descending coronary artery with transthoracic Doppler scan echocardiography. Am Heart J 2002, I43(4):668-75.

7. De Simone L, Caso P, Severino S, D'Andrea A, Mauro C, Monda V, Mininni N: Reduction of coronary flow reserve non-invasively determined by transthoracic Doppler echocardiography as a predictor of left anterior descending coronary artery stenosis. Ital Heart J 2000, I:289-94.

8. Lowenstein J, Tiano C, Presti C, Quiroz C: Noninvasive assessment ofcoronary flow velocity reserve by transthoracic Doppler echocardiography in the everyday echo-laboratory practice experience on 1000 studies. J Am Soc Echocardiogr 2002, I5(Supp):S42.

9. Voci P, Pizzuto F, Mariano E, Puddu PE, Chiavari PA, Romeo F: Measurement of coronary flow reserve in the anterior and posterior descending coronary arteries by transthoracic Doppler ultrasound. Am J Cardiol 2002, 90:988-91.

10. Krzanowski. M, Bodzoń W, Brzostek T, Niżankowski R, Szczeklik A: Value of transthoracic echocardiography for the detection of high grade coronary artery stenosis. Prospective evaluation in 50 consecutive patients scheduled for coronary angiography. J Am Soc Echocardiogr 2000, 13:1091-9. 
II. Krzanowski M, Bodzoń W, Dudek D, Heba G, Rzeszutko M, Niżankowski R, Dubiel J, Szczeklik A: Transthoracic, harmonic mode, contrast enhanced color Doppler echocardiography in detection of restenosis after Percutaneous Coronary Interventions (PCI). Prospective evaluation verified by coronary angiography (accepted for publication by the European Journal of Echocardiography)

12. Clouse M, Cailes C, Devine J, Jordan M, Lester J, Lo L, Shah M, Wenger J, Stevenson G: What is the feasibility of imaging coronary arteries during routine echocardiograms in children? J Am Soc Echocardiogr 2002, 15:1 I27-31.

13. Harada K, Tamura M, Orino T, Yasuoka K: Coronary blood flow assessed by transthoracic echocardiography in neonates. Pediatr Cardiol 2001, 22: 189-93.

14. Harada K, Yasuoka K, Tamura M, Toyono M: Coronary Flow Reserve Assessment by Doppler Echocardiography in Children with and Without Congenital Heart Defect: Comparison with Invasive Technique. I Am Soc Echocardiogr 2002, I5: II $121-6$

15. Di Mario C, Gil R, Serruys PW: Long-term reproducibility of coronary flow velocity measurements in patients with coronary artery disease. Am J Cardiol 1995, 75: I 177-80.

16. Hildick-Smith DJ, Maryan R, Shapiro LM: Assessment of coronary flow reserve by adenosine transthoracic echocardiography: validation with intracoronary Doppler. J Am Soc Echocardiogr 2002, 15:984-90.

17. Ruscazio M, Montisci R, Colonna P, Caiati C, Chen L, Lai G, Cadeddu $M$, Pirisi R, lliceto S: Detection of coronary restenosis after coronary angioplasty by contrast-enhanced transthoracic echocardiographic Doppler assessment of coronary flow velocity reserve. J Am Coll Cardiol 2002, 40:896-903.

18. Dimitrow PP, Krzanowski M, Niżankowski R, Szczeklik A, Dubiel JS: Effect of verapamil on systolic and diastolic coronary blood flow velocity in asymptomatic and mildly symptomatic patients with hypertrophic cardiomyopathy. Heart 2000, 83:262-6.

19. Harada K, Tamura M, Orino T, Yasuoka K: Coronary blood flow assessed by transthoracic echocardiography in neonates. Pediatr Cardiol 2001, 22:189-93.

20. Barata S, Izcovich E, Presti C, Daru V, Grasso E, Sendoya JC, Iglesias $R$ et al: Has sildenafil any effect on coronary flow? Non invasive assessment. J Am Soc Echocardiogr 2002, I (Suppl):S44.

21. Aoki M, Harada K, Yasuoka K, Toyono M, Tamura M: Transthoracic Doppler echocardiographic measurements of flow velocity reserve in left anterior descending coronary artery in children with left ventricular overload secondary to congenital heart disease. J Am Soc Echocardiogr 2002, I 5(Suppl):S72.

22. Hozumi T, Yoshida K, Akasaka T, Asami Y, Kanzaki Y, Ueda Y, Yamamuro A, Takagi T, Yoshikawa J: Value of acceleration flow and the prestenotic to stenotic coronary flow velocity ratio by transthoracic color Doppler echocardiography in noninvasive diagnosis of restenosis after percutaneous transluminal coronary angioplasty. J Am Coll Cardiol 2000, 35: I64-8.

Publish with Bio Med Central and every scientist can read your work free of charge

"BioMed Central will be the most significant development for disseminating the results of biomedical research in our lifetime. "

Sir Paul Nurse, Cancer Research UK

Your research papers will be:

- available free of charge to the entire biomedical community

- peer reviewed and published immediately upon acceptance

- cited in PubMed and archived on PubMed Central

- yours - you keep the copyright

Submit your manuscript here:

http://www.biomedcentral.com/info/publishing_adv.asp
BioMedcentral 